\title{
Detecting dark matter WIMPs in the Draco dwarf: A multiwavelength perspective
}

\author{
Sergio Colafrancesco, ${ }^{1, *}$ Stefano Profumo, ${ }^{2, \dagger}$ and Piero Ullio ${ }^{3, \dagger}$ \\ ${ }^{1}$ INAF-Osservatorio Astronomico di Roma, via Frascati 33, I-00040 Monteporzio, Italy \\ and Istituto Nazionale di Fisica Nucleare, Sezione di Roma 2, I-00133 Roma, Italy \\ ${ }^{2}$ Division of Physics, Mathematics and Astronomy, California Institute of Technology, \\ Mail Code 106-38, Pasadena, California 91125, USA \\ ${ }^{3}$ Scuola Internazionale Superiore di Studi Avanzati, Via Beirut 2-4, I-34014 Trieste, Italy \\ and Istituto Nazionale di Fisica Nucleare, Sezione di Trieste, I-34014 Trieste, Italy
}

(Received 3 July 2006; published 11 January 2007)

\begin{abstract}
We explore the possible signatures of dark matter pair annihilations in the nearby dwarf spheroidal galaxy Draco. After investigating the mass models for Draco in the light of available observational data, we carefully model the dark matter density profile, taking advantage of numerical simulations of hierarchical structure formation. We then analyze the gamma-ray and electron/positron yield expected for weakly interacting dark matter particle (WIMP) models, including an accurate treatment of the propagation of the charged particle species. We show that unlike in larger dark matter structures — such as galaxy clusters - spatial diffusion plays here an important role. While Draco would appear as a pointlike gamma-ray source, synchrotron emission from electrons and positrons produced by WIMP annihilations features a spatially extended structure. Depending upon the cosmic ray propagation setup and the size of the magnetic fields, the search for a diffuse radio emission from Draco can be a more sensitive indirect dark matter search probe than gamma rays. Finally, we show that available data are consistent with the presence of a black hole at the center of Draco: if this is indeed the case, very significant enhancements of the rates for gamma rays and other emissions related to dark matter annihilations are expected.
\end{abstract}

DOI: 10.1103/PhysRevD.75.023513

\section{INTRODUCTION}

The astrophysical search for signals of dark matter (DM) particle pair annihilations in cosmic structures on large scales (from galaxies to clusters of galaxies) is, potentially, a very powerful technique, highly complementary to direct DM searches, in the quest for the identification of the fundamental nature of DM. The widest and more definite set of results can be harvested through a multifrequency survey of DM annihilation signals over the whole electromagnetic (e.m.) spectrum (see, e.g. [1], hereafter CPU2006, and references therein) by using a detailed treatment of both the microscopic interaction properties of the hadronic and leptonic secondary yields of WIMP annihilations, and of the subsequent emissions originating by the yields themselves in the astrophysical environment at hand. Various astrophysical systems have been taken into consideration to this aim. The central regions of ordinary galaxies (like our own Galaxy) are usually considered among the best places to set constraints on the presence and on the nature of DM particles (see, e.g, [2] for a review, and the analyses in [3-5], among others).

The typical faintness of DM signals within viable WIMP scenarios makes, in fact, the galactic center, or the central regions of nearby galaxies (like M31), the most plausible and promising places to detect signals of WIMP annihilations. However, the expected DM signals have to contend, there, with the rich and often poorly understood astrophys-

\footnotetext{
*Electronic address: colafrancesco@mporzio.astro.it

${ }^{\dagger}$ Electronic address: profumo@caltech.edu

Electronic address: ullio@sissa.it
}

PACS numbers: 95.35.+d, 12.60.Jv, 98.56.Wm, 98.70.Rz

ical context of thermal and nonthermal sources ( $\mathrm{SN}$ remnants, pulsars, molecular clouds, to mention a few), whose spectral energy distributions (SEDs) cover the whole e.m. spectrum, reaching even $\mathrm{TeV}$ energy scales, as the recent results from HESS, MAGIC, and Cangaroo have clearly shown (see, e.g., [6] and references therein; see, however, also [7]). In this respect, galaxy cores are likely not the best places to definitely identify DM annihilation signals.

Galaxy clusters have the advantage to be massdominated by DM and, in some cases, like the nearby Coma cluster, to have a quite extended spectral and spatial coverage of thermal and nonthermal emission features which enable to set interesting constraints on the properties of DM (see, e.g., $[1,8,9]$ and references therein for various aspects of the DM SEDs in clusters). The study of the DMinduced SEDs in galaxy clusters has been shown to be quite constraining for DM WIMP models, and can even be advocated to shed light on some emission features (e.g., radio halos, hard-X-ray and UV excesses, and gamma-ray emission) which are still unclear. Nonetheless, the sensitivity and spatial resolution of the present and planned experiments in the gamma rays, $\mathrm{x}$ rays, and radio do not likely allow to probe more than a few nearby clusters. It is therefore mandatory to remain within the local environment to have reasonable expectations to detect sizable emission features of possible DM signals.

Globular clusters have also been proposed (see e.g., [10]) as possible sources of gamma rays from WIMP annihilations, but with expected signals well below the sensitivity threshold of future experiments, mainly due to their quite low mass-to-light ratios. 
The ideal astrophysical systems to be used as probes of the nature of DM should be mostly dark (i.e., dominated by $\mathrm{DM}$ ), as close as possible (in order to produce reasonably high fluxes), and featuring central regions mostly devoid of sources of diffuse radiation at radio, x-rays frequencies, and gamma-rays frequencies, where the DM SEDs peak (see, e.g. CPU2006 for general examples).

Dwarf spheroidal (dSph) galaxies closely respond to most of these requirements, as they generally consist of a stellar population, with no hot or warm gas, no cosmic-ray population, and little or no dust (see, e.g., [11] for a review). Several $\mathrm{dSph}$ galaxies populate the region around the Milky Way and M31, and some of them seem to be dynamically stable and featuring high concentrations of DM.

Among these systems, the Draco dSph is one of the most interesting cases. This object has already been considered as a possible gamma-ray source fed by DM annihilations in recent studies [12-16], in part triggered by an anomalous excess of photon counts from Draco reported by the CACTUS collaboration in a drift-scan mode survey of the region surrounding the $\mathrm{dSph}$ galaxy [17]. The nature of the effect is still controversial, but it has been shown in $[14,15]$ to be in conflict, in most WIMP models, with the EGRET null-result in the search for a gamma-ray source from the direction of Draco [18]. Other gamma-ray upper limits have been obtained by the Whipple 10-m telescope collaboration as well (see e.g., [19]).

The observational state-of-the-art for Draco goes, however, beyond gamma-ray emissions: radio continuum upper limits on Draco have been obtained by Fomalont et al. [20] with the VLA. These authors report an upper limit of $J_{\nu}<2 \mathrm{mJy}$ at $\nu=4.9 \mathrm{GHz}$ (this is a $3 \sigma$ level limit). Typical magnetic field strength of $B \sim 2-4 \mu \mathrm{G}$ for dwarf galaxies similar to Draco have also been derived from radio observations at $5 \mathrm{GHz}$ [21]. The $\mathrm{x}$-ray emission from the central part of Draco has an upper limit provided by ROSAT [22]. The count rate detected by the PSPC instrument in the $(0.1-2.4) \mathrm{keV}$ energy band is $<0.9 \times$ $10^{-3} \mathrm{~s}^{-1}$ corresponding to an unabsorbed flux limit of $F_{X}<1.7 \times 10^{-14} \mathrm{erg} \mathrm{cm}^{-2} \mathrm{~s}^{-1}$. This flux corresponds to an X-ray luminosity upper limit of $L_{X}<0.01 \times$ $10^{36} \mathrm{erg} \mathrm{s}^{-1}$.

The main point we wish to make in the present analysis is that a complete multifrequency analysis of the astrophysical DM signals coming from Draco might carry much more information, and can be significantly more constraining, in terms of limits on DM WIMP models than, for instance, a study of the emissions in the gamma-ray frequency range alone.

As we show in the present analysis, available observational data, and the possible detection of WIMP annihilation signals from Draco by future instruments can be, in principle, of crucial relevance for the study of the nature of WIMP DM: the expected emission features associated to
DM annihilation secondary products are, in fact, the only radiation mechanisms which can be expected in a system like a dSph, as originally envisioned by Colafrancesco $[9,23]$. Following our original suggestions, and pursuing the systematic approach we outlined for the case of Coma (see CPU 2006), we present here a detailed analysis and specific predictions for the WIMP DM annihilation signals expected from Draco in a multiwavelength strategy.

Specifically, we first derive the DM density profile of Draco in a self-consistent $\Lambda$ CDM scenario in Sec. II. We then discuss the gamma-ray emission produced in Draco from DM annihilation, assuming a set of modelindependent WIMP setups [1]. Gamma-ray emissions, and constraints, are studied in Sec. III. We then present in Sec. IV the signals expected from Draco at all frequencies covered by the radiation originating from the secondary products: synchrotron emission in the radio range, inverse Compton scattering of electrons and positrons produced by DM annihilation off cosmic microwave background (CMB) and starlight photons, and the associated Sunyaev Zel'dovich (SZ) effect. We also discuss in Sec. V the possible amplification of these signals by an intervening black hole at the center of Draco. We present our conclusions in the final Sec. VI.

Throughout this paper, we refer to the concordance cosmological model suggested by WMAP 3 yr. [24]; namely, we assume that the present matter energy density is $\Omega_{m}=0.266$, that the Hubble constant in units of $100 \mathrm{~km} \mathrm{~s}^{-1} \mathrm{Mpc}^{-1}$ is $h=0.71$, that the present mean energy density in baryons is $\Omega_{b}=0.0233 / h^{2}$, with the only other significant extra matter term in cold dark matter $\Omega_{\mathrm{CDM}}=\Omega_{m}-\Omega_{b}$, that our Universe has a flat geometry and a cosmological constant $\Lambda$, i.e. $\Omega_{\Lambda}=1-\Omega_{m}$, and, finally, that the primordial power spectrum is scale invariant and is normalized to the value $\sigma_{8}=0.772$.

\section{THE DARK MATTER DENSITY PROFILE IN DRACO}

Modeling the distribution of dark matter for dSph's is not a straightforward task. The radial maps of the star velocity dispersions clearly indicate that $\mathrm{dSph}$ are dark matter dominated systems. However, available observational data do not provide enough information to unequivocally determine the shape and concentration of the supporting dark matter density profiles (see e.g. the recent analysis of Ref. [25] for the case of Draco, under investigation here). Such freedom is partially reduced restricting to $\Lambda \mathrm{CDM}$ inspired scenarios, as appropriate for dark matter in the form of cold WIMP particles. Within this structure formation picture, numerical N-body simulations of hierarchical clustering predict that Milky Way size galaxies contain an extended population of substructures, with masses extending down to the free streaming scale for the CDM component (as small as $10^{-12}-10^{-3} M_{\odot}$ in the case of neutralinos in supersymmetric models or in other WIMP 
setups [26,27]), and surviving, at least in part, to tidal disruption: dwarf satellites stand as peculiar objects, since they are the smallest ones featuring a stellar counterpart, while mechanisms preventing star formation are supposed to intervene for lighter objects (among scenarios supporting this interpretation, see, e.g., [28]). In case of isolated CDM halos, properties of the dark matter density profile have been investigated in some detail through numerical simulations: a universal shape and a correlation (on average) between the object mass and its concentration are expected (more details will be given in the following section). The picture is less clear for satellites, like Draco, standing well within the dark matter potential well of the hosting halo. Tidal forces may have significantly remodeled the internal structure of these objects, an effect which is likely to depend, e.g., upon the merging history of each satellite. Based again on numerical simulations, significant departures from the correlation between the mass and concentration parameter observed for isolated halos have been reported in the literature, as well as discrepant results regarding whether the universal shape of the density profile is preserved [29] or not [30] in the subhalos, after tides have acted and these systems have reached a new equilibrium configuration.

\section{A. Mass models within the $\Lambda \mathrm{CDM}$ framework}

The main dynamical constraint we consider for mass models for the Draco dSph is the observed line-of-sight velocity dispersion of its stellar population. The underlying, necessary, assumption we shall make here is that the stellar component is in equilibrium, and hence that the Jeans equation applies to this system; if this is the case, one finds that the projection along the line of sight (1.o.s.) of the radial velocity dispersion of stars can be expressed in terms of $M(r)$, the total (i.e. including all components) mass within the radius $r$ [31,32]:

$$
\begin{aligned}
\sigma_{\mathrm{los}}^{2}(R)= & \frac{2 G}{\sum(R)} \int_{R}^{\infty} \mathrm{d} r^{\prime} \nu\left(r^{\prime}\right) M\left(r^{\prime}\right)\left(r^{\prime}\right)^{2 \beta-2} \int_{R}^{r^{\prime}} \mathrm{d} r \\
& \times\left(1-\beta \frac{R^{2}}{r^{2}}\right) \frac{r^{-2 \beta+1}}{\sqrt{r^{2}-R^{2}}}
\end{aligned}
$$

where $\nu(r)$ is the density profile of the stellar population and $\Sigma(R)$ represents its surface density at the projected radius $R$. In the derivation of Eq. (1), we have assumed that the anisotropy parameter $\beta$ is constant over the radius; in terms of the radial and tangential velocity dispersion, respectively $\sigma_{r}$ and $\sigma_{\theta}, \beta=1-\sigma_{\theta}^{2} / \sigma_{r}^{2}: \beta=1$ denotes the case of purely radial orbits, $\beta=0$ that of a system with isotropic velocity dispersion, while $\beta \rightarrow-\infty$ labels circular orbits. As we will see shortly, the anisotropy parameter is important since we recover in our analysis the wellknown degeneracy between the reconstructed mass profile and the assumed degree of stellar anisotropy.
Extensive photometric studies are available for the Draco dwarf; we refer to the analysis in Odenkirchen et al. [33] relying on multicolor data from the Sloan Digital Sky Survey (SDSS) (sample and foreground determination labeled S2 in that analysis) and reproduce the result for the radial profile of the surface brightness in Fig. 1 (left panel). We also show two alternative fits of the data: one option is the generalized exponential profile proposed by Sersic [34] and implemented in the case of Draco also by Lokas, Mamon, and Prada [35]:

$$
\Sigma(R)=\Sigma_{0} \exp \left[-\left(R / R_{\mathrm{S}}\right)^{1 / m}\right],
$$

choosing the parameter $1 / m=1.2$, and fitting the scale radius $R_{\mathrm{S}}$ and central surface brightness $\Sigma_{0}$ to the data (the best-fit procedure gives $R_{\mathrm{S}}=7^{\prime} .3$ ). As second possibility, we follow Mashchenko et al. [25] and consider a modified Plummer model:

$$
\Sigma(R)=\Sigma_{0}\left[1+\left(R / R_{\mathrm{P}}\right)^{2}\right]^{-(\alpha-1) / 2},
$$

setting the exponent $\alpha=7$, and then fitting the value for the scale radius $\left(R_{\mathrm{P}}=14^{\prime} .6\right)$. For each of the two $\Sigma(R)$, the luminosity density profile $\nu(r)$ is obtained by inverting the definition of surface brightness with the Abel integral formula, i.e. implementing the deprojection:

$$
\nu(r)=-\frac{1}{\pi} \int_{r}^{\infty} d R \frac{1}{\sqrt{R^{2}-r^{2}}} \frac{d \Sigma}{d R} .
$$

The inversion is performed numerically for the Sersic profile, while it can be done analytically for the modified Plummer model; results are shown in Fig. 1 (right panel) and one can see that the mild differences in the surface brightness are only marginally amplified in the luminosity density profiles. Here we are referring to luminosities in the V-band and, following again [35], we have adopted for the distance of Draco the value $80 \mathrm{kpc}$ [36], or, equivalently, a distance modulus of 19.5 [37], standing in between (and in agreement at $1 \sigma$ ) the other recent estimate of $75.8 \pm$ $0.7 \pm 5.4 \mathrm{kpc}$ from Ref. [38] and the value of $82 \pm 6 \mathrm{kpc}$ from the compilation of Mateo [39]. To add the stellar component in the total mass term $M(r)$ in Eq. (1), we need an estimate for the stellar mass-to-light ratio in the V-band; we mainly refer to one of the largest values quoted in the literature, $\Upsilon_{V}=3 M_{\odot} / L_{\odot}$, including in it a possible subdominant gas component.

The ansatz we implement for the dark matter component is that of a spherical distribution sketched by a radial density profile:

$$
\rho(r)=\rho^{\prime} g(r / a) ;
$$

given in terms of the function $g(x)$ and of two parameters, i.e. a scale radius $a$ and a normalization factor $\rho^{\prime}$. This is in analogy with the usual description of dark matter halos from results of numerical N-body simulations in terms of a universal density profile; we take as a guideline for our mass models the form originally proposed by Navarro, 

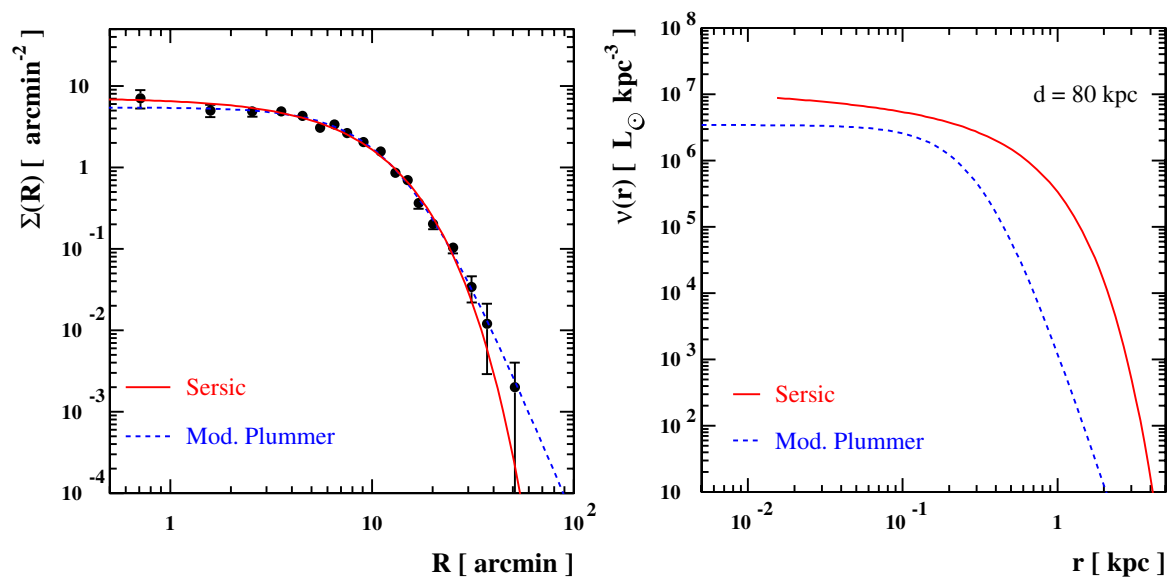

FIG. 1 (color online). Left panel: radial profile for the surface brightness distribution of stars in Draco; data are from Odenkirchen et al. [33], while fits are with a Sersic profile or a modified Plummer model. Right panel: the corresponding luminosity density profiles.

Frenk, and White [40]:

$$
g_{\mathrm{NFW}}(x)=\frac{1}{x(1+x)^{2}}
$$

and a shape slightly more singular towards the center proposed by Diemand et al. [41] (hereafter labeled as D05 profile):

$$
g_{D 05}(x)=\frac{1}{x^{\gamma}(1+x)^{3-\gamma}} \quad \text { with } \quad \gamma \simeq 1.2 .
$$

As a further option, we consider the Burkert profile [42]:

$$
g_{B}(x)=\frac{1}{(1+x)\left(1+x^{2}\right)}
$$

i.e. a model with a large core, in agreement with the gentle rise in the inner part of the rotation curves occurring in a vast class of galaxies, including dwarfs [42,43]. Mechanisms of gravitational heating of the dark matter by baryonic components during or after the baryon infall have been advocated to reconcile these observations with the central density cusps of the profiles introduced above [44-46]; these models are still contrived and it is probably premature to say whether in the case of Draco a cored or cuspy halo is expected.

Since we shall extrapolate the dark matter mass profile well beyond the radial size of the stellar component, we need a description of the regime where the profile gets sensibly reshaped by tidal interactions with the dark matter halo of the Milky Way. We compute the tidal radius $r_{\text {tid }}$ in the impulse approximation, as appropriate for extended objects $[47,48]$ :

$$
\frac{M\left(r_{\text {tid }}\right)}{r_{\text {tid }}^{3}}=\left.\left[2-\frac{r}{M_{M W}(r)} \frac{\partial M_{M W}}{\partial r}\right] \frac{M_{M W}(r)}{r^{3}}\right|_{r=r_{p}-r_{\text {tid }}}, g f
$$

with $M\left(r_{\text {tid }}\right)$ the mass of Draco within the tidal radius, and $M_{M W}(r)$ the mass of the Milky Way within the galactocen- tric distance $r$; the expression on the right-hand side is computed for the orbital radius of Draco $r_{p}$ at its latest pericenter passage.

Mass models for Draco are generated as follows: for a given functional form for the profile and for any given pairs of the parameters $\rho^{\prime}$ and $a$, the density profile is shifted into the form [49]:

$$
\rho(r) \rightarrow \rho(r) \exp \left(-r / r_{\text {tid }}\right)
$$

with $r_{\text {tid }}$ determined from Eq. (9), assuming for the Milky Way a virial mass equal to $10^{12} M_{\odot}$ and a Navarro Frenk White (NFW) profile with concentration parameter equal to 13 [50]; $r_{p}$ will be taken, as a first test case, equal to $20 \mathrm{kpc}$, which is about the minimum pericenter radius below which tidal effects would be visible in the stellar component as well [25], and which gives the most conservative estimate for the dark matter mass in Draco. We are then ready to implement Eq. (1) and compare against data.

Munoz et al. [51] have recently made a novel compilation of 1.o.s. star velocity dispersions in Draco, containing 208 stars; they show results implementing several binning criteria, among which we resort to the one with the largest number of stars per bin (21 stars per bin), which is the least susceptible to statistical fluctuations. Using essentially the same data sample, but a different binning, Wilkinson et al. [52] find a sharp drop in the velocity dispersion corresponding to the bin at the largest circular radius, a feature that does not emerge in the analysis of Munoz et al. On the other hand, Lokas, Mamon, and Prada [35] question whether this sample should be further cleaned from outliers, i.e. stars that may not actually be gravitationally bound to Draco. We will compare separately with the data set from Munoz et al., i.e. in 10 bins out to a circular radius of slightly larger than $30^{\prime}$, and the one from Wilkinson et al., i.e. 7 bins out to a circular radius of about $35^{\prime}$, see Fig. 2. For any mass model we consider the reduced $\chi^{2}$ variable: 


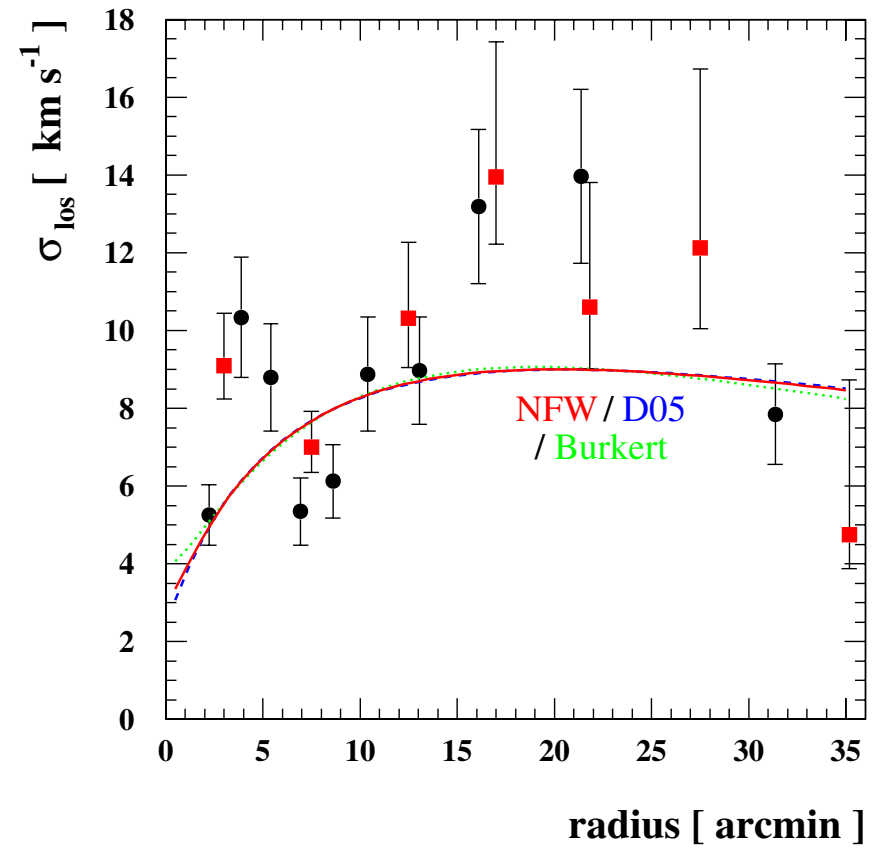

FIG. 2 (color online). Line-of-sight velocity dispersion for the three best-fit models considered in the paper. Data are from Munoz et al., 2005 (filled circles) and from Wilkinson et al. (filled squares).

$$
\chi_{\text {red }}^{2}=\frac{1}{N_{\text {bins }}} \sum_{j=1}^{N_{\text {bins }}} \frac{\left(\sigma_{\text {los }}\left(R_{j}\right)-\sigma_{\text {los }}^{j}\right)^{2}}{\left(\Delta \sigma_{\text {los }}^{j}\right)^{2}} .
$$

$\chi_{\text {red }}^{2}$ is very sensitive to the value of the overall normalization parameter, moderately sensitive to $\beta$, while it is less sensitive to the length scale $a$. In Fig. 2 we show the lineof-sight velocity dispersion projected along the l.o.s., com-

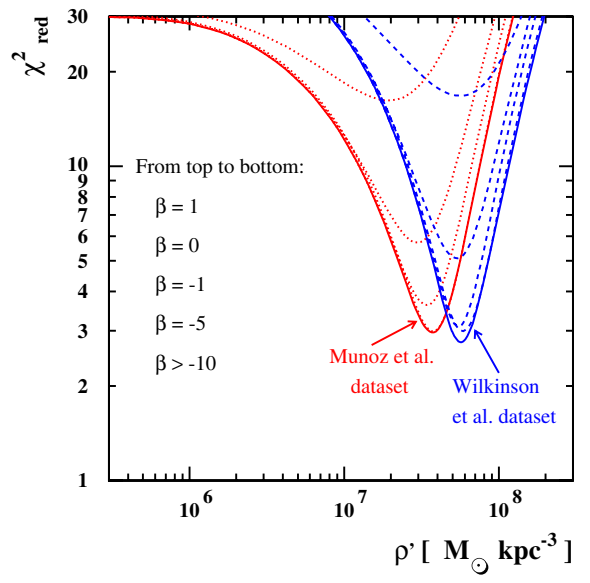

paring to the Munoz et al. data set and assuming the star distribution according to the Sersic profile. The best-fit models for the three dark matter density profiles we are focusing on are set as follows: (i) a NFW profile with $a=$ $1 \mathrm{kpc}, \rho^{\prime}=3.710^{7} M_{\odot} \mathrm{kpc}^{-3}, r_{\text {tidal }}=1.7 \mathrm{kpc}$, and $\beta=$ -3.7; (ii) a Burkert profile with $a=0.5 \mathrm{kpc}, \rho^{\prime}=$ $2.110^{8} M_{\odot} \mathrm{kpc}^{-3}, r_{\text {tidal }}=2.0 \mathrm{kpc}$, and $\beta=-1.0$; (iii) a D05 profile with $a=1 \mathrm{kpc}, \rho^{\prime}=2.5410^{7} M_{\odot} \mathrm{kpc}^{-3}$, $r_{\text {tidal }}=1.5 \mathrm{kpc}$, and $\beta=-6.3$. Clearly, the data set does not allow for a discrimination among the three models.

In Fig. 3 we illustrate the sensitivity of the fit to some of the parameters introduced in our model, taking the NFW profile as a reference case, and $a=1 \mathrm{kpc}$ as in the best-fit model: the minimum of $\chi^{2}$ is well defined with respect to $\rho^{\prime}$ and has a marginal shift when comparing to the data as in the binning of Wilkinson et al.; had we followed the suggestion of Ref. [35] to take out of the sample some of the stars that appear as outlier, the minimum reduced $\chi^{2}$ would get below 1 , but its position on the $\rho^{\prime}$ axis would not change appreciably. Also shown is the dependence of the result upon the anisotropy parameter $\beta$ : for the NFW profile, the case of radial orbits is disfavored, while models with circular anisotropy give better fits. In the right panel of Fig. 3 we show instead that none of our additional assumptions have a significant impact on the velocity dispersion fit. In particular, there is a marginal effect when considering an alternative fit to the stellar profile, or when varying the assumed value for the distance of the Draco within the ranges of estimates quoted in the literature, or when decreasing the mass-to-light ratio of the stellar component to significantly smaller values. Also secondary, but slightly larger, is the effect of assuming that the current position of Draco is also the smallest

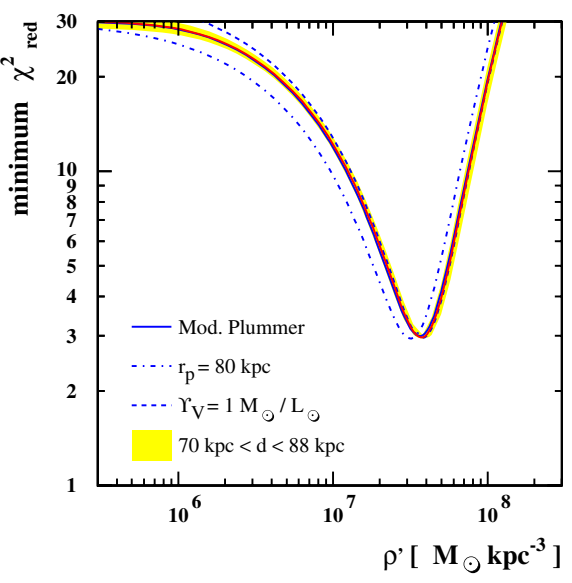

FIG. 3 (color online). We consider the NFW profile as a reference halo model, fix the length scale parameter to $1 \mathrm{kpc}$, and plot the reduced $\chi^{2}$ as a function of the density normalization parameter $\rho^{\prime}$. In the left panel we plot the reduced $\chi^{2}$ obtained either for the Munoz et al. or Wilkinson et al. data sets, for a few selected values of the anisotropy parameter $\beta$ or choosing the parameter $\beta$ (in the interval $-10<\beta<1$ ) which at a given $\rho^{\prime}$ gives the smallest reduced $\chi^{2}$; other underlying assumptions (default model) are: radial star profile described by a Sersic profile, distance at latest pericenter passage $r_{p}=20 \mathrm{kpc}$, mass-to-light ratio of $3 M_{\odot} / L_{\odot}$, and distance of Draco $d=80 \mathrm{kpc}$. In the right panel we show that none of these latter assumptions are crucial: we plot as a function of $\rho^{\prime}$ the minimum reduced $\chi^{2}$ for $\beta$ between -10 and 1 , in the case of radial star profile according to the modified Plummer model, $r_{p}$ increased to $80 \mathrm{kpc}$, mass-to-light ratio decreased to $1 M_{\odot} / L_{\odot}$, and the distance of Draco varied within a generous range of values. 

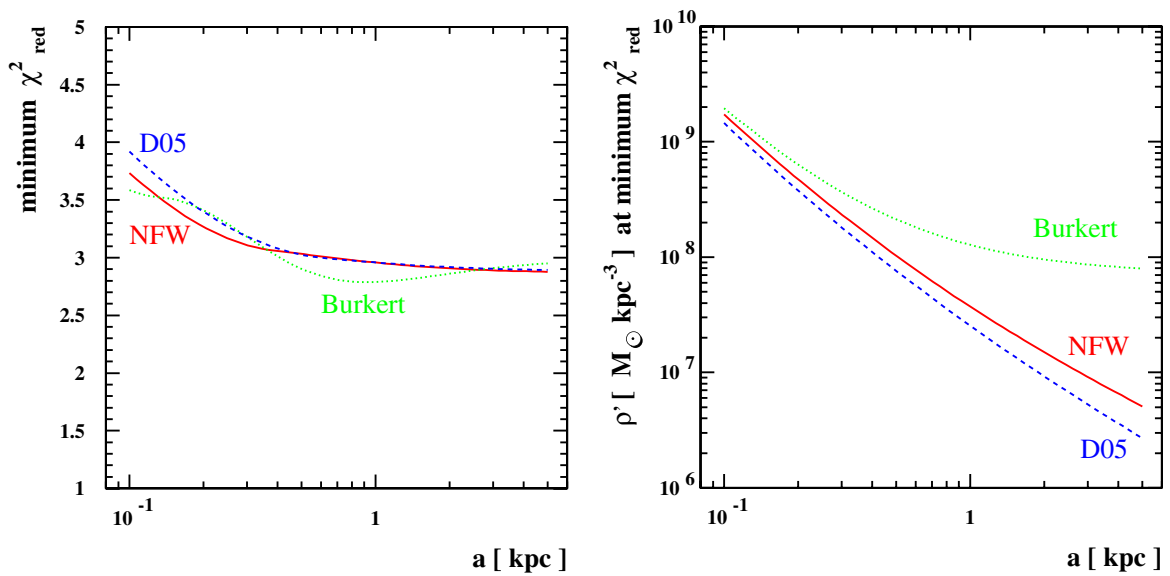

FIG. 4 (color online). Left panel: for a given value of the length scale parameter $a$, we plot the minimum $\chi_{\text {red }}^{2}$ obtained by varying the parameter $\rho^{\prime}$ and the anisotropy parameter $\beta$ between -10 and 1; we consider the three dark matter halo profiles introduced in the text, compare against the Munoz et al. data set, and refer to our default model for the other parameters. Right panel: for a given value of the length scale parameter $a$, value of $\rho^{\prime}$ corresponding to the minimum $\chi_{\text {red }}^{2}$ displayed in the left panel for the three dark matter halo profiles.

galactocentric distance reached so far in its orbital motion, and hence it is the relevant radius to estimate the effects of tidal stripping (in this case, tidal radii become much larger than the scale radius for the stellar component).

In Fig. 4 we show the minimum value of the reduced $\chi^{2}$, obtained taking the density normalization $\rho^{\prime}$ and the stellar anisotropy $\beta$ as free parameters, for the three dark matter density profiles and as a function of the scale factor $a$ : as clearly emerging from the figure, the data set does not allow for a clear discrimination in the parameter $a$, but there is, rather, a close correlation between the length scale and density normalization parameter. In the right panel of Fig. 4 we plot the value of $\rho^{\prime}$ corresponding to the model with minimum $\chi^{2}$ and a given scale factor $a$; note the huge span in the range of values of the logarithmic vertical scale.
In Fig. 5 we show the tidal radii as determined assuming for the radius at the last pericenter passage $20 \mathrm{kpc}$ or $80 \mathrm{kpc}$ (right panel), and values of $\beta$ (left panel) set as in the bestfit models; shallower, or less concentrated, profiles give equivalent fits to the data if the degree in circular anisotropy is decreased $(\beta=-10$ is the minimum value we are scanning on; isotropic, $\beta=0$, models are favored for the cored Burkert profile in the case of moderate to large values for the scale factor).

\section{B. Connections to the structure formation picture}

The possibility of discriminating among dark matter halo models increases when we take into account results from N-body simulations of structure formation. To make
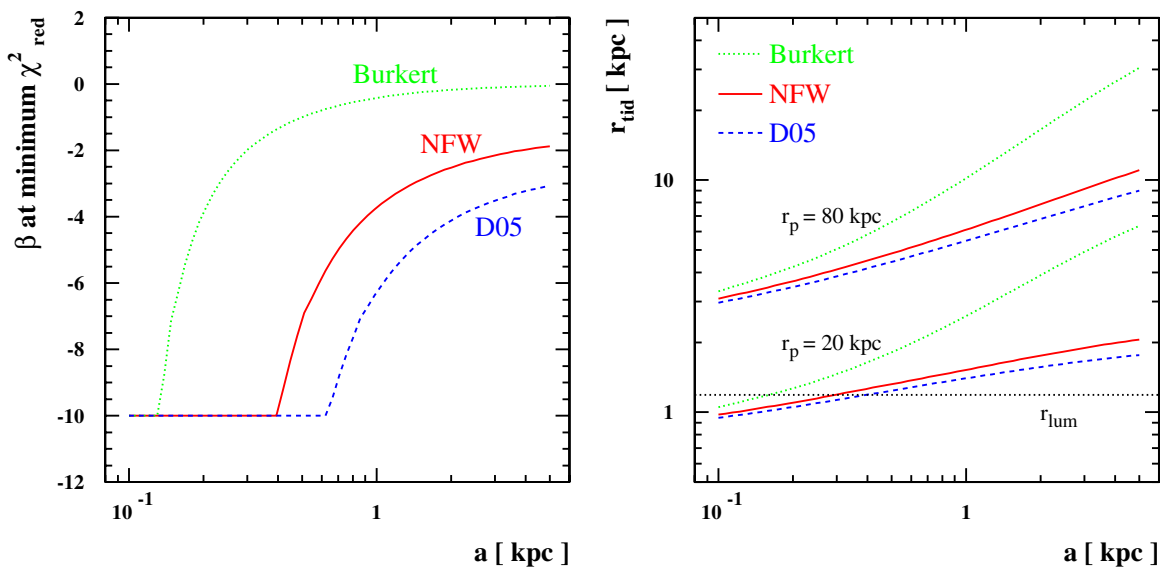

FIG. 5 (color online). Values of $\beta$ (left panel) and of the tidal radius (right panel, lower curves corresponding to a radius at latest pericenter passage equal to $20 \mathrm{kpc}$ ) defining the model with minimum $\chi_{\text {red }}^{2}$ at a given $a$ displayed in the left panel of Fig. 4, for the three dark matter density profiles. We also show in the right panel the value of the tidal radius setting the radius at latest pericenter passage to $80 \mathrm{kpc}$, and keeping other parameters unchanged; values for $\chi_{\text {red }}^{2}$ change marginally, i.e. from the point of view of fitting the data the two cases are equivalent, but obviously all $r_{\text {tid }}$ increase significantly (for reference we plot with a dotted horizontal line the radial size of the stellar component). 
this step we need, however, to rely on a series of extrapolations. The first is to try to map the fit we made for a tidally disrupted object, well within the Milky Way potential well, to the configuration of a virialized system, unaffected by tides, of the kind described, on statistical grounds, by results of simulations. We refer to the prescription derived from numerical studies in [48]: let the density profile prior to tidal interactions be in the form:

$$
\rho_{\text {no tides }}(r)=\rho_{s} g\left(r / r_{s}\right) \text {. }
$$

Suppose, then, that tidal interactions change it into the form:

$$
\rho(r)=f_{t} \exp \left(-r / r_{\text {tid }}\right) \rho_{\text {no tides }}(r),
$$

assuming that the length scale parameter $a$ in the final profile is equal to the initial scale factor $r_{s}$. Comparing the form of Eq. (10) to the one we used in the fit to the stellar velocity dispersion, i.e. Eq. (13), we find $\rho^{\prime}=f_{t} \rho_{s}$. The parameter $f_{t}$ is a dimensionless measure of the reduction in central density due to tidal effects; simulations indicate that the latter is correlated to the mass fraction of the satellite bound to the object after the effect of tides, $m_{\text {bnd }}$, through the expression [48]:

$$
\begin{aligned}
\log f_{t}= & -0.007+0.35 \log m_{\text {bnd }}+0.39\left(\log m_{\text {bnd }}\right)^{2} \\
& +0.23\left(\log m_{\text {bnd }}\right)^{3}
\end{aligned}
$$

(we will assume this phenomenological fitting formula to be valid for $m_{\text {bnd }}$ larger than about 5\%). According to this scheme, we can uniquely assign to any best-fit model with given $\rho^{\prime}$ and $a$ (for an assumed pericenter radius through which, $r_{\text {tid }}$ is determined) the corresponding $\rho_{s}$ and $r_{s}$, or equivalently a value for the initial virial mass of the object $M_{\mathrm{vir}}$ and its concentration parameter $c_{\mathrm{vir}}$, defined as $c_{\mathrm{vir}}=$ $R_{\mathrm{vir}} / r_{-2}$. In this last step we introduced the virial radius

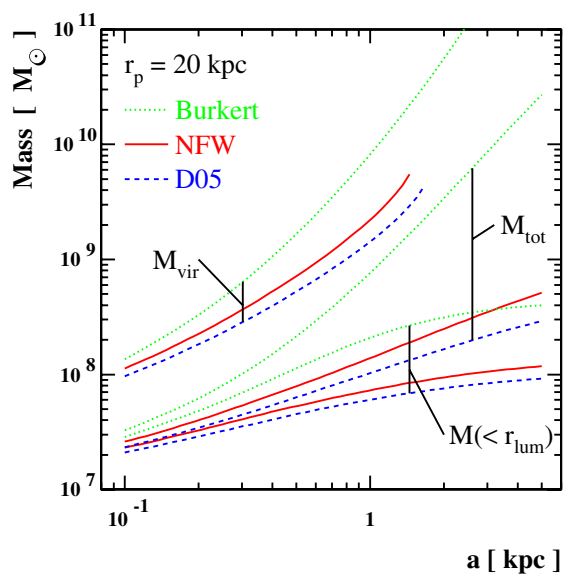

$R_{\text {vir }}$, defined as the radius within which the mean density of the halo is equal to the virial overdensity $\Delta_{\text {vir }}(\simeq 340$ at $z=0$ ) times the mean background density, and the radius $r_{-2}$ where the effective logarithmic slope of the density profile is $-2\left(r_{-2}\right.$ is equal to $a$ for the NFW profile, $0.8 a$ for the D05, and about $1.5 a$ for the Burkert profile). In Fig. 6 we plot $M_{\text {vir }}$ for the best-fit models displayed in Figs. 4 and 5; for comparison, we also show the total halo mass bound to Draco after tidal stripping, and the dark matter mass within the spherical shell defined by the radius of the stellar component, i.e. $r_{\text {lum }}=51^{\prime}$. We have referred to the two extreme choice of pericenter radii, i.e. $20 \mathrm{kpc}$ and $80 \mathrm{kpc}$; the procedure seems fairly consistent since in the two cases we get very close values for $M_{\text {vir }}$ (in the case of a small pericenter radius and the NFW or D05 profile, at large $a$ the fraction of mass loss becomes very large and extrapolations according to Eq. (14) become unreliable, so values of $M_{\text {vir }}$ are not displayed). In Fig. 7 we plot, for the same best-fit models, the concentration parameter versus virial mass; we also show the $M_{\mathrm{vir}}-c_{\mathrm{vir}}$ correlation as extrapolated, for the currently preferred cosmological setup [53], from the toy model of Bullock et al. [54], which is tuned to reproduce the scaling found in numerical simulations for isolated halos. As far as substructures are concerned, concentrations are expected to be systematically larger, since substructures form, on average, in a denser environment with respect to isolated halos; for illustrative purposes only, we show the $M_{\text {vir }}-c_{\text {vir }}$ scaling in the case of a $50 \%$ and a $100 \%$ increase in concentration.

We have already stressed a few times that our analysis is heavily relying on extrapolations, so no firm conclusion can be derived; nevertheless, our results seem to indicate that we should prefer models with an intermediate $M_{\text {vir }}$, say $10^{9} M_{\odot}$, corresponding to $a$ of the order of $1 \mathrm{kpc}$ for the NFW and D05 profiles and about $0.5 \mathrm{kpc}$ for the Burkert

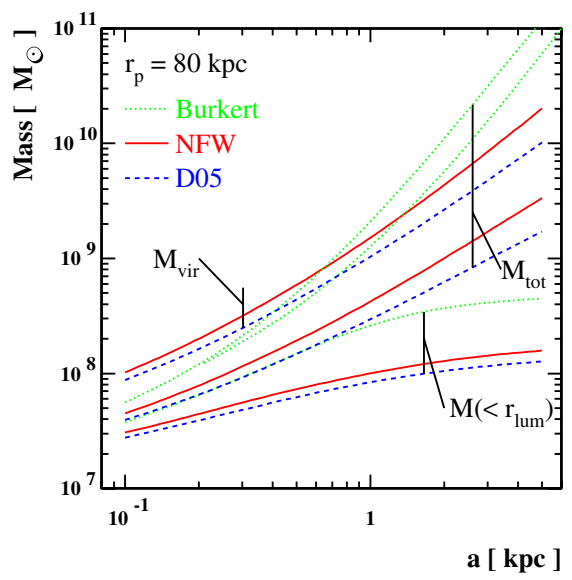

FIG. 6 (color online). We display, for the minimum $\chi_{\text {red }}^{2}$ models illustrated in Figs. 4 and 5, values of the dark matter halo mass within the radial size of stellar component (lower curves) and of the total mass in the dark matter component for the calculated tidal radius (medium curves), assuming a pericenter radius of $20 \mathrm{kpc}$ (left panel) and $80 \mathrm{kpc}$ (right panel). We have also performed an extrapolation to estimate the initial virial mass of Draco, i.e. the mass associated to it before sinking deep into the potential well of the Milky Way and the loss of a large fraction of such initial mass due to tidal effects. 


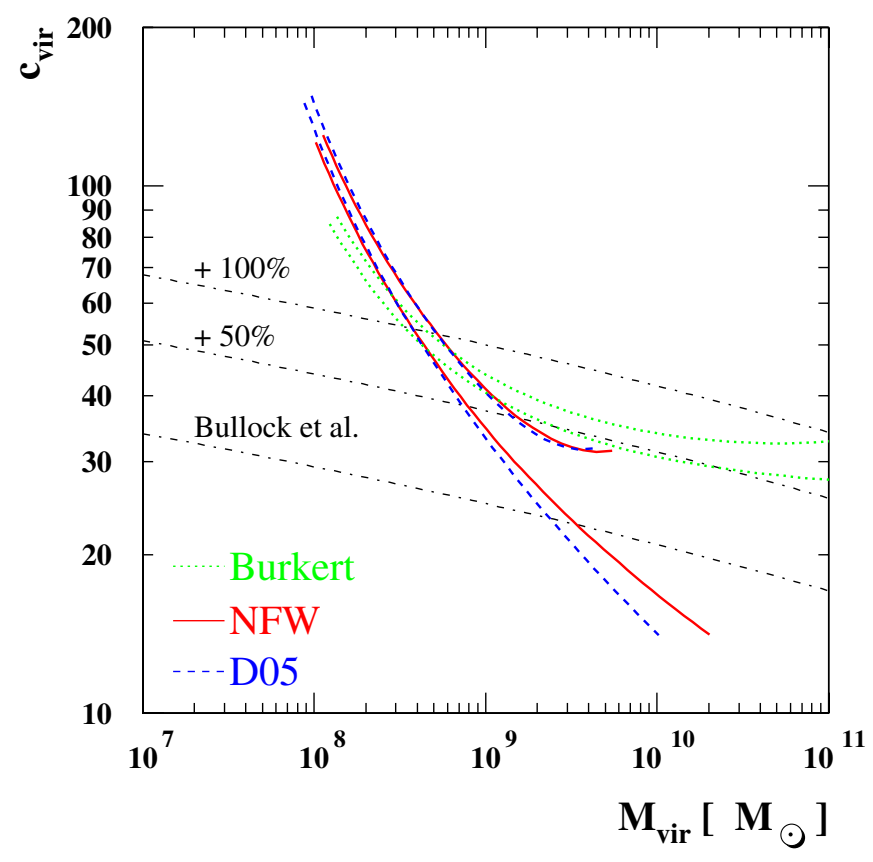

FIG. 7 (color online). We display, for the minimum $\chi_{\text {red }}^{2}$ models illustrated in Figs. 4 and 5, the extrapolated values of the virial mass, and the concentration parameter according to the prescription for the response to tidal interactions introduced in [48] and fitted to numerical simulations. Also shown is the extrapolation with the Bullock et al. prescription of the correlation $M_{\text {vir }}-c_{\text {vir }}$ for isolated halos, assuming a $50 \%$ or a $100 \%$ increase in concentration in the case of subhalos.

profile (such cases are those that have been chosen as reference models in Fig. 2) and that the range of length scale values allowed in Figs. 4 and 5 is probably a very generous one, with values at the lower and upper ends which should be most likely dropped. The range of models we are indicating here as preferred by the NFW profile is analogous to the one suggested in Ref. [25], although the two approaches differ. In particular we will not implement here a constraint from the age of Draco stellar population which is used as a guideline in [25]: to do that we would need to build a subhalo mass function for the Milky Way matching the observed satellite pattern, and to model star formation within subhalos, two steps which are not very well understood and on which the degree of extrapolation would be inevitably much more drastic than what we have accepted so far.

\section{THE GAMMA-RAY SIGNAL FROM WIMP ANNIHILATIONS IN DRACO}

WIMPs have a small but finite probability to annihilate in pairs, giving rise to potentially observable standard model yields. Two ingredients intervene in fixing source functions: on the one hand, the annihilation cross section, branching ratios, and spectral distributions for the yields are specified in any given particle physics scenario embed- ding the WIMP; on the other hand, source functions scale with the number density of WIMP pairs, i.e. in the case we are considering here, they are proportional to the square of the dark matter mass density in Draco. Since photons in the energy range we are interested to, i.e. smaller than few $\mathrm{TeV}$, propagate through the interstellar medium without being absorbed, predictions for the induced gamma-ray fluxes are straightforward and simply involve an integral of the source along the line of sight; the expression for the flux per unit energy and solid angle, is usually cast in the form:

$$
\phi_{\gamma}\left(E_{\gamma}, \Theta, \Delta \Omega\right)=\frac{(\sigma v)}{8 \pi M_{\chi}^{2}} \sum_{f} \frac{d N_{\gamma}^{f}}{d E}(E) B_{f} J(\Theta, \Delta \Omega),
$$

where $(\sigma v)$ is the WIMP annihilation rate at zero temperature, $M_{\chi}$ the WIMP mass, and the sum is over all kinematically allowed annihilation final states $f$, each with a branching ratio $B_{f}$. It is beyond the scope of the present analysis to review the ranges of values and the modeldependent determination of these parameters, as well as of the gamma-ray spectral distributions $d N_{\gamma}^{f} / d E$, topics which have been vastly discussed in recent literature; we will mainly refer here either to a toy model in which we pick particular values for $M_{\chi}$ and $(\sigma v)$, and assume to have only one dominant annihilation channel: it is useful to consider the case of a soft annihilation channel such as a $b-\bar{b}$ pair, and to contrast it with the hard $\tau^{+}-\tau^{-}$final state. As we showed in [1], these toy models are well justified in the context of solidly motivated theoretical grounds, for instance within the paradigm of neutralino dark matter. For definiteness, and for illustrative purposes, we shall also make use of special, well-studied, benchmark supersymmetric models, as we did for the case of our analysis of the multiwavelength emissions from Coma in Ref. [1].

In Eq. (15) the dependence on the halo profile has been factorized out defining:

$$
J(\Theta, \Delta \Omega)=\frac{1}{\Delta \Omega} \int_{\Delta \Omega} d \Omega \int_{\text {l.o.s. }} d l \rho^{2}(l),
$$

where $\Theta$ is the direction of observation and the average is over the angular acceptance (or the angular resolution) of the detector $\Delta \Omega$. In Fig. 8 we plot the range of the expected values for $J$ towards the center of Draco, for two sample values of $\Delta \Omega$ and within the minimum $\chi^{2}$ halo models selected in the previous section. Confirming other recent analyses $[13,55,56]$, our results show that there is a very small spread in the prediction for $J$, even referring to significantly different dark matter halo shapes and even for small angular acceptances: within a factor of a few and in units of $\mathrm{GeV}^{2} \mathrm{~cm}^{-6} \mathrm{kpc}, J$ is about 100 for $\Delta \Omega=$ $10^{-5} \mathrm{sr}$ and about 1 for $\Delta \Omega=10^{-3} \mathrm{sr}$. Such a small spread is in contrast to what one finds in the analogous estimate when considering the Milky Way galactic center 


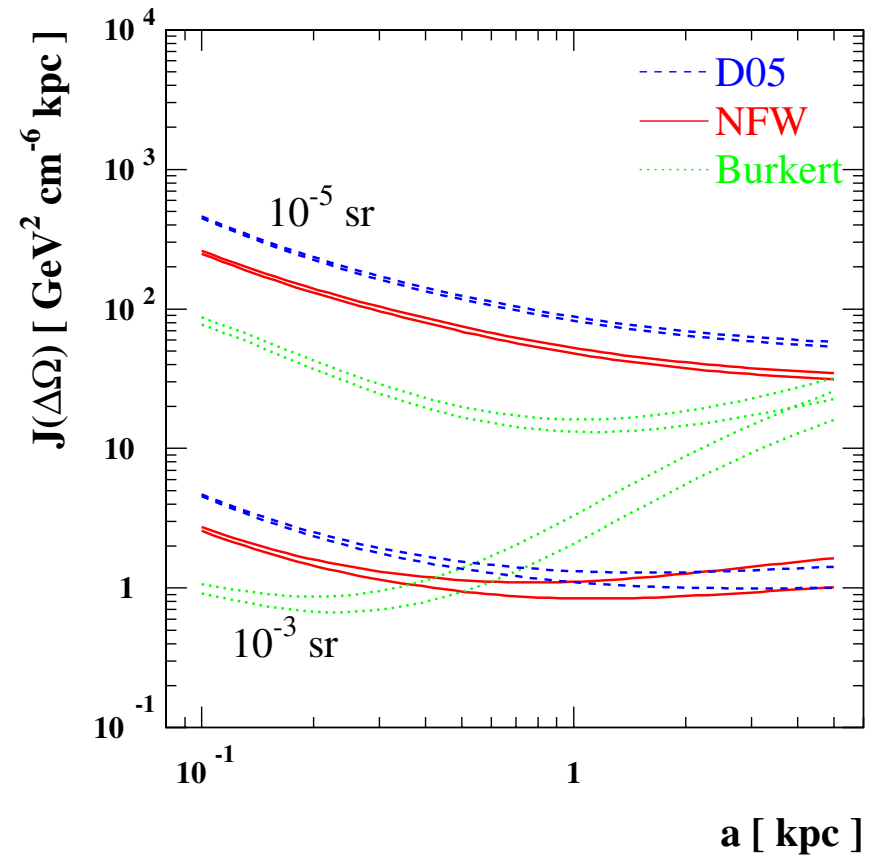

FIG. 8 (color online). We display, for the minimum $\chi_{\text {red }}^{2}$ models illustrated in Figs. 4 and 5, the line-of-sight integral function $J$, towards the center of Draco and for two different angular acceptance $\Delta \Omega$.

as a source of gamma rays from dark matter annihilation. One can apply the same procedure of fitting different halo profiles to the Milky Way dynamical constraints and then extrapolate their radial scalings down to the innermost parsec or so; the focus is on the eventual sharp dark matter density enhancement which could be present in the galactic center region: for singular profiles the values of $J$ one derives may be very large, up to about $10^{4}-10^{5}$ for NFW profiles and $\Delta \Omega=10^{-5} \mathrm{sr}$ (see, e.g. [13]; note however that in Ref. [13] a dimensionless $J$ is adopted and to translate values quoted therein into those for the definition adopted here, one should scale them by the factor $0.765 \mathrm{GeV}^{2} \mathrm{~cm}^{-6} \mathrm{kpc}$ ), but drop dramatically, with a decrease as large as 4 orders of magnitude, when considering less singular or cored profiles. In the case of Draco, the distance of the source is much larger and the l.o.s. integral involves an average over a large volume, smoothing out the effect of a singularity in the density profile; at the same time, however, the mean dark matter density is on average fairly large for any profile, since the dark halo concentration parameter is large.

In its all-sky survey, EGRET has accumulated a limited exposure towards Draco. A report on the collected data is given in [18]; the analysis aims at the identification of a point source at the center of Draco; seven energy bins are considered, each with the appropriate angular cuts, no point source is found, and the photon counts are consistent with the expected flux from diffuse emission, except for a 2 event "excess" in the energy range between 1 and $10 \mathrm{GeV}$, with a total of 6 events found versus 4.1 expected in the standard background scenario (notice that no statistical evidence for such excess is claimed in [18] or in the present analysis). In Fig. 9 we show, for a given WIMP mass, the value of the annihilation cross section required for a flux matching the 2 events in EGRET between 1 and $10 \mathrm{GeV}$, for exposures and angular cuts as specified in the data analysis, assuming our reference NFW best-fit halo model and $b-\bar{b}$ (left panel) or $\tau^{+}-\tau^{-}$(right panel) annihilation channels. Also shown in the figure are expected sensitivity curves with GLAST, the next gamma-ray telescope in space, and for upcoming observations of Draco with ground-based ACT telescopes.

Regarding the GLAST detector, we refer to an updated simulation of the instrument performance [57]: we refer to the energy dependent sensitivities of the two large area telescope (LAT) sections, the thin (or front) section of the tracker (peak effective area above $1 \mathrm{GeV}$ of about $5500 \mathrm{~cm}^{2}, 68 \%$ containment angle varying between $0.6 \mathrm{deg}$ at $1 \mathrm{GeV}$ and $0.04 \mathrm{deg}$ at $100 \mathrm{GeV}$ ) and of the thick (or back) section of the tracker (peak effective area above $1 \mathrm{GeV}$ of about $4500 \mathrm{~cm}^{2}, 68 \%$ containment angle varying between $1 \mathrm{deg}$ at $1 \mathrm{GeV}$ and $0.07 \mathrm{deg}$ at $100 \mathrm{GeV}$ ). To estimate the background, we include an extragalactic component at the level found in EGRET data [58], extrapolated to higher energy with a $E^{-2.1}$ power law, plus a galactic component scaling like $E^{-2.7}$ (such scaling is expected from the decay of $\pi^{0}$ generated by the interaction of primary protons with the interstellar medium; we are neglecting an eventual inverse Compton (IC) component, since, if present, such a term is most likely already included as misidentified extragalactic) and normalized in such way that, together with the extragalactic component, it gives the 6 events above $1 \mathrm{GeV}$ detected by EGRET (assuming just for 4.1 events for the background level does not change significantly our projected sensitivities). We consider a 5 yr exposure time, in an all-sky survey mode for which the effective area in the direction of Draco is, on average, about $30 \%$ of the peak effective area (area when the source is at the zenith of the instrument). Finally, we define a $\chi^{2}$ variable as:

$$
\chi^{2}=\sum_{j=1}^{n_{\text {bins }}} \frac{N s_{j}^{2}}{N s_{j}+N b_{j}},
$$

where $N s_{j}$ and $N b_{j}$ stand for the number of signal and background events in each bin, restricting to bins with more than 5 signal events. The bin selection should in principle be optimized model by model; in general we find that it is a good choice to take three bins per decade in energy (two or more bins are grouped into one in case this procedure gives 5 signal events; this sometimes happens in the highest energy bin included in the sum above), while at any given energy we integrate over a solid angle which is the largest between the point spread function (PSF) set by the $68 \%$ containment angle (full energy dependence included for each section of the tracker) and 

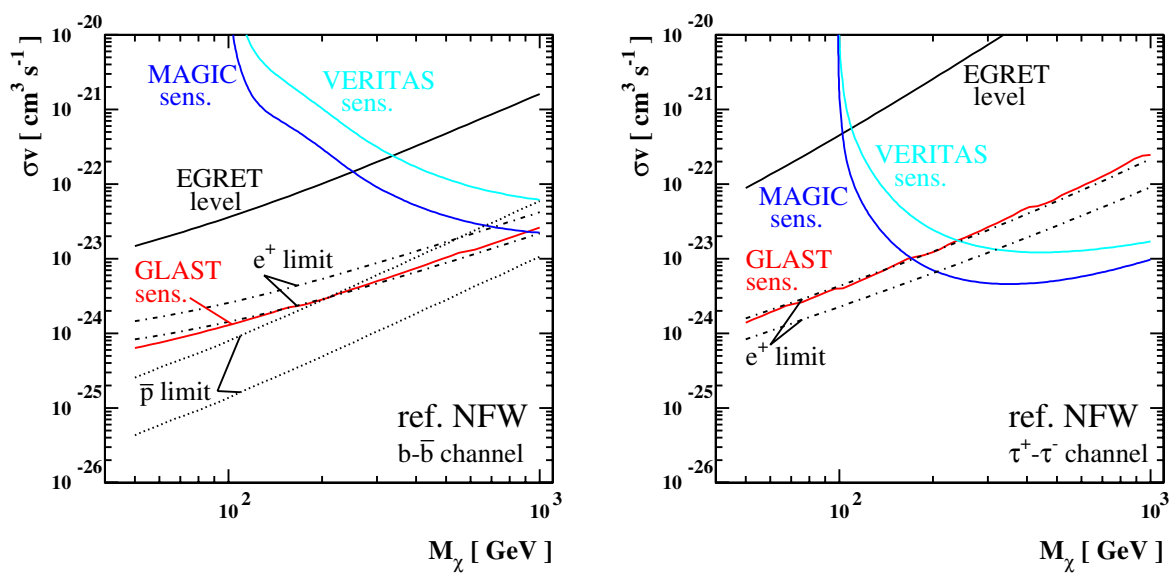

FIG. 9 (color online). We show the WIMP mass - WIMP pair annihilation cross section plane, assuming a $b-\bar{b}$ (left) and $\tau^{+}-\tau^{-}$ (right) dominant annihilation final state. The solid black line indicates the value of the annihilation cross section required for a flux matching the 2 events in EGRET between 1 and $10 \mathrm{GeV}$, for exposures and angular cuts as specified in the data analysis, assuming our reference NFW best-fit halo model; the black dotted and dot-dashed lines show the limits from the flux of, respectively, antiprotons and positrons in the Milky Way halo (the two lines corresponding to two different cosmic-ray propagation setups); the solid curves indicate the projected sensitivities of GLAST, MAGIC, and VERITAS (see the text for more details).

the solid angle which maximizes the ratio $\Phi_{s} / \sqrt{\Phi_{s}+\Phi_{b}}$. Sensitivity curves are given in Fig. 9 as $3 \sigma$ discovery limits; the latter are found to be, with the present accurate modeling of the detector, slightly less promising than the analogous curves obtained in other recent estimates $[55,56]$.

Regarding Air Cherenkov Telescopes (ACTs), we consider the detection prospects with instruments in the northern hemisphere, i.e. MAGIC [59], which is currently taking data, and VERITAS, which will be completed soon extending the current single mirror telescope to an array of at first four, then later seven telescopes [60]. We assess the discovery sensitivity of the two ACTs using a low energy threshold of $100 \mathrm{GeV}$, and the effective collection area as a function of energy recently quoted by the two collaborations in Refs. [59,60]; the main sources of background for ACTs correspond to misidentified gammalike hadronic showers and cosmic-ray electrons. The diffuse gammaray background gives a subdominant contribution to the background, which we also took into account using the same figures outlined above for the space-based telescopes background. We use the following estimates for the ACT cosmic-ray background [61]:

$$
\begin{aligned}
\frac{\mathrm{d} N_{\text {had }}}{\mathrm{d} \Omega}\left(E>E_{0}\right)= & 6.1 \times 10^{-3} \epsilon_{\mathrm{had}}\left(\frac{E_{0}}{1 \mathrm{GeV}}\right)^{-1.7} \\
& \times \mathrm{cm}^{-2} \mathrm{~s}^{-1} \mathrm{sr}^{-1}, \\
\frac{\mathrm{d} N_{\mathrm{el}}}{\mathrm{d} \Omega}\left(E>E_{0}\right)= & 3.0 \times 10^{-2}\left(\frac{E_{0}}{1 \mathrm{GeV}}\right)^{-2.3} \mathrm{~cm}^{-2} \mathrm{~s}^{-1} \mathrm{sr}^{-1},
\end{aligned}
$$

where $\epsilon_{\text {had }}$ parametrizes the efficiency of hadronic rejection, which we assume to be at the level of $10 \%$ [59,60]. As above, we proceed with an optimized binning of the energy range of interest (extending from the energy threshold up to the WIMP mass), compute the number of signal and background events in each bin, and require that the resulting $\chi^{2}$ (evaluated according to the analogue of Eq. (17)) gives a statistical excess over background.

The models for which we predict a detectable flux have fairly large cross sections, still compatible but in the high end of models with a thermal relic density, as computed in a standard cosmological scenario, which matches the observed dark matter density in the Universe [53], see, e.g., [1] (another possibility is that we refer to models with nonthermal relic components, such as from the decay of moduli fields, or to modified cosmological setups affecting the Hubble parameter at the time of WIMP decoupling, see, e.g., [62]). Large annihilation cross sections give enhanced signals for any indirect dark detection technique, in particular, we need to check whether this picture is compatible with the antimatter fluxes measured at Earth: in fact, pair annihilation of WIMP in the halo of the Milky Way is acting as a source of primary positrons and antiprotons which diffuse in the magnetic field of the Galaxy, building up into exotic antimatter populations. Current measurements of the local antiproton flux are consistent with the standard picture of antiprotons being secondary particles generated by the primary cosmic-ray protons in spallation processes [63]; on the other hand, a weak evidence of an excess in the positron flux has been claimed [64,65], in a picture that is going to become increasingly clearer with the ongoing measurements in space by the recently launched Pamela detector [66]. We estimate the induced flux of positrons and antiprotons (no antiprotons are generated in the $\tau^{+}-\tau^{-}$channel), referring to the same Milky Way halo model we have introduced to estimate tidal effects on Draco, and to the diffusive convective model for the propagation of charged 
particles implemented in the DarkSUSY package [67]. Parameters in the propagation model are chosen in analogy with a standard setup [68] in the GALPROP propagation package [69], or the most conservative choice suggested in Ref. [70] which can still reproduce ratios of secondaries to primaries as measured in cosmic-ray data while minimizing the flux induced by WIMP annihilations: these give, respectively, the lower and upper curves plotted in Fig. 9 and corresponding to the $3 \sigma$ limits on the annihilation cross section obtained by comparing the WIMP-induced fluxes to a full compilation of present data on the local antiproton and positron fluxes. The values displayed should not be taken as strict exclusion curves, since we are not doing a full modeling of the uncertainties in the propagation model, nor scanning on more general configurations of the Milky Way dark matter halo; relaxing them by a factor of 2 to 5 or maybe even larger should be relatively straightforward; they can be however taken as a guideline to see that models in such a region of the parameter space should be testable with higher precision antimatter data, while models at the EGRET level are most probably already excluded by current data.

Finally in Fig. 10 we show how flux levels and projected sensitivities scale scanning over our sample of halo models for Draco, for a sample WIMP mass and the $b-\bar{b}$ final state; antiproton limit levels are also plotted for comparison.

This work which is based on realistic estimates of both the dark matter density distribution in Draco and of the

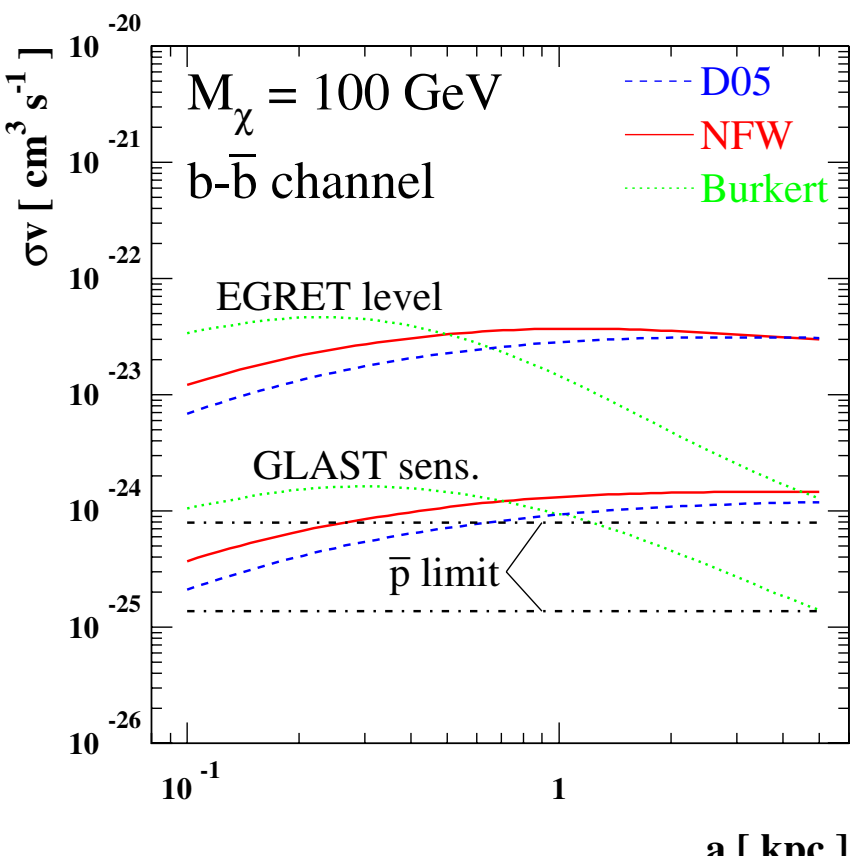

FIG. 10 (color online). As in Fig. 9, but keeping the WIMP mass fixed at $100 \mathrm{GeV}$, and varying the halo scale length parameter $a$ for the three dark matter density profiles considered here.
WIMP physical set up leaves the early Cactus claims (see, e.g., $\quad$ http://ucdems.ucdavis.edu/solar2/results/ Chertok.PANIC05.pdf) of a detection of gamma-ray emission from Draco apart.

\section{MULTIWAVELENGTH SIGNALS FROM DRACO}

The following step is to extend our analysis to the radiation emitted at lower frequencies. For this purpose, we need to track the injection of electrons and positrons from WIMP annihilations in Draco, as well as their propagation in space and energy; it will then be possible to make predictions for the induced synchrotron and inverse Compton radiations. Our starting point is the assumption that, in analogy to more massive objects such as the Milky Way itself, there is a random component of interstellar magnetic fields associated with Draco and that it is a fair approximation to model the propagation of charged particles as a diffusive process. In this limit we can calculate the electron and positron number densities implementing to the following transport equation:

$$
\begin{aligned}
\frac{\partial}{\partial t} \frac{d n_{e}}{d E}= & \nabla\left[D(E, \vec{x}) \nabla \frac{d n_{e}}{d E}\right]+\frac{\partial}{\partial E}\left[b(E, \vec{x}) \frac{d n_{e}}{d E}\right] \\
& +Q_{e}(E, \vec{x}),
\end{aligned}
$$

where $Q_{e}$ is the electron or positron source function from WIMP annihilations:

$$
Q_{e}(E, \vec{x})=\frac{1}{2 M_{\chi}^{2}} \sum_{f} \frac{d N_{e}^{f}}{d E}(E) B_{f} \rho^{2}(\vec{x}),
$$

while $D$ is the diffusion coefficient and $b$ the energy loss term. In Ref. [1] we derived the analytic solution to this equation in case of a spherical symmetric system and for $D$ and $b$ that do not depend on the spatial coordinates. We refer here to a time-independent source and consider the limit for an electron number density that has already reached equilibrium; the solution takes the form:

$$
\frac{d n_{e}}{d E}(r, E)=\frac{1}{b(E)} \int_{E}^{M_{\chi}} d E^{\prime} \hat{G}\left(r, v-v^{\prime}\right) Q_{e}\left(r, E^{\prime}\right)
$$

with:

$$
\begin{aligned}
\hat{G}(r, \Delta v)= & \frac{1}{[4 \pi(\Delta v)]^{1 / 2}} \sum_{n=-\infty}^{+\infty}(-1)^{n} \int_{0}^{r_{h}} d r^{\prime} \frac{r^{\prime}}{r_{n}} \\
& \times\left[\exp \left(-\frac{\left(r^{\prime}-r_{n}\right)^{2}}{4 \Delta v}\right)-\exp \left(-\frac{\left(r^{\prime}+r_{n}\right)^{2}}{4 \Delta v}\right)\right] \\
& \times \frac{\rho^{2}\left(r^{\prime}\right)}{\rho^{2}(r)} .
\end{aligned}
$$

In the Green function $\hat{G}$ the energy dependence has been hidden in two subsequent changes of variable $v=$ $\int_{u_{\min }}^{u} d \tilde{u} D(\tilde{u})$ and $u=\int_{E}^{E_{\max }} \frac{d E^{\prime}}{b\left(E^{\prime}\right)}$; the radial integral extends up to the radius of the diffusion zone $r_{h}$ at which a free escape boundary condition is imposed, as enforced by 
the sum over $n$, having defined $r_{n}=(-1)^{n} r+2 n r_{h}$. Whenever the scale of mean diffusion $\sqrt{\Delta v}$, covered by an electron while losing energy from energy at the source $E^{\prime}$ to the energy when interacting $E$, is much smaller than the scale over which $\rho^{2}$ has a significant variation, $\hat{G}$ is close to 1, and spatial diffusion can be neglected; in Ref. [1] it was shown that this limit applies in the case of the Coma cluster. For dSph we find that, most likely, we are in the opposite regime.

To model electron and positron energy losses we choose as a reference value for the magnetic field $B_{\mu}=1 \mu \mathrm{G}$ and a thermal electron density of $10^{-6} \mathrm{~cm}^{-3}$ : these values are derived from radio observations of dwarf galaxies similar to Draco at $5 \mathrm{GHz}$ (Klein et al. 1992) and from the assumption that the ROSAT PSPC x-ray upper limit on Draco (Zang and Meurs, 2001) is due to thermal bremsstrahlung, respectively. For the diffusion coefficient we assume the Kolmogorov form $D(E)=D_{0} / B_{\mu}^{1 / 3}(E / 1 \mathrm{GeV})^{1 / 3}$, fixing the constant $D_{0}=3 \times 10^{28} \mathrm{~cm}^{2} \mathrm{~s}^{-1}$ in analogy with its value for the Milky Way; finally our guess for the dimension of the diffusion zone is that, again consistently with the picture relative to the Milky Way, $r_{h}$ is about twice the radial size of the luminous component, i.e., here, 102 arcmin (we will refer to this set of propagation parameters as set \#1). In the left panel of Fig. 11 we show that, in this setup, electrons and positrons lose a moderate fraction of their energies on scales $\sqrt{\Delta v}$ that are comparable to the size of the diffusion region, i.e. spatial diffusion as a large effect. Even referring to an extreme model in which the diffusion coefficient is decreased by 2 orders of magni- tudes down to $D_{0}=3 \times 10^{26} \mathrm{~cm}^{2} \mathrm{~s}^{-1}$ (this would imply a much smaller scale of uniformity for the magnetic field), adding on top of that a steeper scaling in energy, $D(E)=$ $D_{0}(E / 1 \mathrm{GeV})^{-0.6}$ (this is the form sometimes assumed for the Milky Way; we label this propagation parameter configuration set \#2), scales $\sqrt{\Delta v}$ are decreased but remain still relatively large. In the right panel of Fig. 11 we consider a WIMP model with mass $100 \mathrm{GeV}$ annihilating in the $b \bar{b}$ final state within our reference NFW halo model for Draco. We show integrals over volume within the radial coordinate $r$ of the equilibrium number density $d n_{e} / d E$, for a few values of the energy $E$, and for the set of propagation parameters \#1 and \#2, as well as the results corresponding to the assumption that spatial diffusion can be neglected. All integrals are normalized to the integrals over the whole diffusion region of $d n_{e} / d E$ for the corresponding energy $E$ and assuming negligible spatial diffusion: we deduce from the figure that for set \#1 there is a depletion of the electron/positron populations with a significant fraction leaving the diffusion region, while for set \#2 they are more efficiently confined within the diffusion region but still significantly misplaced with respect to the emission region.

For a given electron/positron equilibrium distribution we can infer the induced synchrotron and inverse Compton emissions. In the limit of frequency $\nu$ of the emitted photons much larger than the nonrelativistic gyro-frequency $\nu_{0}=e B /(2 \pi m c) \simeq 2.8 B_{\mu} \mathrm{Hz}$, the spontaneously emitted synchrotron power takes in the form [71]:

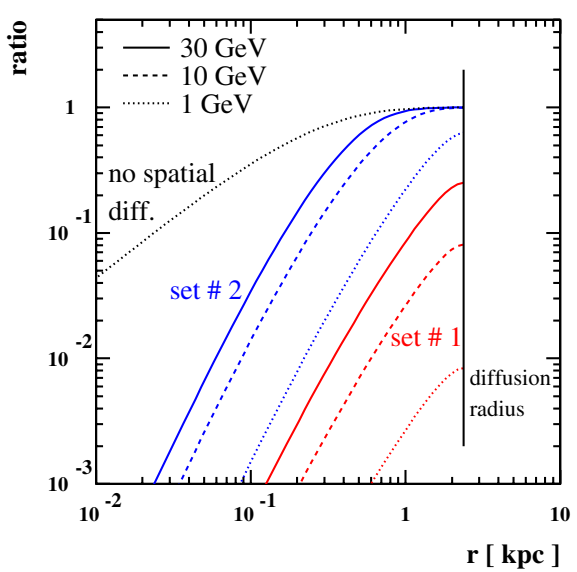

FIG. 11 (color online). Left panel: mean diffusion distance $\sqrt{\Delta v}$ covered by an electron while losing energy from its energy at emission $E^{\prime}$ to its energy at interaction $E$; three different $E^{\prime}$ are considered, as well as a conservative choice for propagation parameters (labeled by "set \#1" in the plot) or a more extreme choice (labeled by "set \#2" in the plot), see the text for details. Right panel: we plot, as a function of the distance from the center of Draco $r$, the integral over volume up the radius $r$ of the electron number density $d n_{e} / d E(r, E)$, for three values of the energy $E$ and for the two choices of propagation parameters already considered in the left panel; also shown is the case when $d n_{e} / d E$ is computed assuming negligible spatial diffusion. For each energy, integrals over volume are normalized to the integral over volume up to the assumed radius of diffusion for Draco of $d n_{e} / d E$ in the limit in which spatial diffusion is neglected; we have chosen the reference NFW model for the dark halo, and a WIMP of mass $100 \mathrm{GeV}$ annihilating into $b \bar{b}$. As can be seen, compared to the case when spatial diffusion is neglected, in the actual cases applying to Draco there is a sharp deficit of electrons in the inner region of even within the total diffusion volume. 
$P_{\text {synch }}(\nu, E, r)=\int_{0}^{\pi} d \theta \frac{\sin \theta}{2} 2 \pi \sqrt{3} r_{0} m c \nu_{0} \sin \theta F(x / \sin \theta)$,

where we have introduced the classical electron radius $r_{0}=e^{2} /\left(m c^{2}\right)=2.82 \times 10^{-13} \mathrm{~cm}$, and we have defined the quantities $x$ and $F$ as:

$$
x \equiv \frac{2 \nu}{3 \nu_{0} \gamma^{2}}\left[1+\left(\frac{\gamma \nu_{p}}{\nu}\right)^{2}\right]^{3 / 2},
$$

and

$$
F(t) \equiv t \int_{t}^{\infty} d z K_{5 / 3}(z) \simeq 1.25 t^{1 / 3} \exp (-t)\left[648+t^{2}\right]^{1 / 12}
$$

Folding the synchrotron power with the spectral distribution of the equilibrium number density of electrons and positrons, we find the local emissivity at the frequency $\nu$ :

$$
j_{\text {synch }}(\nu, r)=\int_{m_{e}}^{M_{\chi}} d E\left(\frac{d n_{e^{-}}}{d E}+\frac{d n_{e^{+}}}{d E}\right) P_{\text {synch }}(\nu, E, r) .
$$

In Fig. 12 we consider a reference WIMP model with a mass of $100 \mathrm{GeV}$, annihilating into $b-\bar{b}$ with a cross section at the level to induce a gamma-ray flux matching the 2 events in EGRET between 1 and $10 \mathrm{GeV}$ for a dark matter distribution as in our reference NFW model. In the left panel we plot the radio flux density spectrum integrated over the whole diffusion volume:

$$
S_{\text {synch }}(\nu)=\int d^{3} r \frac{j_{\text {synch }}(\nu, r)}{4 \pi d^{2}},
$$

with $d$ the distance of Draco. The spectrum is significantly flatter for the propagation parameter set \#1, since the peak in emitted photon frequency is linearly proportional to the energy of the emitting particle, and electrons and positrons tend to escape from the diffusion box while losing energy, rather than remaining confined within it and giving a signal which piles up at lower frequencies. We then introduce the azimuthally averaged surface brightness distribution:

$$
I_{\text {synch }}(\nu, \Theta, \Delta \Omega)=\int_{\Delta \Omega} d \Omega \int_{\text {l.o.s. }} d l \frac{j_{\text {synch }}(\nu, l)}{4 \pi},
$$

where the integral is performed along the l.o.s. $l$, within a cone of angular acceptance $\Delta \Omega$ centered in a direction forming an angle $\Theta$ with the direction of the center of Draco. In the right panel of Fig. 12 we plot surface brightness integrated over a cone of 3 arcsec width, corresponding to the tiny angular acceptance of the VLA at the time it was used to perform searches for point radio sources in the central 4 arcmin of Draco [20]; no source was found and the corresponding upper limit is plotted in the figure. To illustrate how the shape of the signal changes compared to that of the source function, we also plot the surface brightness which we would obtain in the limit of no spatial diffusion. In Ref. [72] radio fluxes are computed in this limit and the VLA measurement is exploited to exclude WIMP models; we have demonstrated in our discussion that the limit of no spatial diffusion is not likely to hold in the case of Draco and the figure illustrates the fact that, with the present data, limits on the model stemming from radio frequencies are less constraining than in the gammaray band. The figure illustrates also another point: the gamma-ray flux retraces the WIMP annihilation source function; in the example we have considered, even with the angular resolution at which future observations will be carried out, Draco would appear as a single point source. On the other hand, in the radio band the signal is spread out

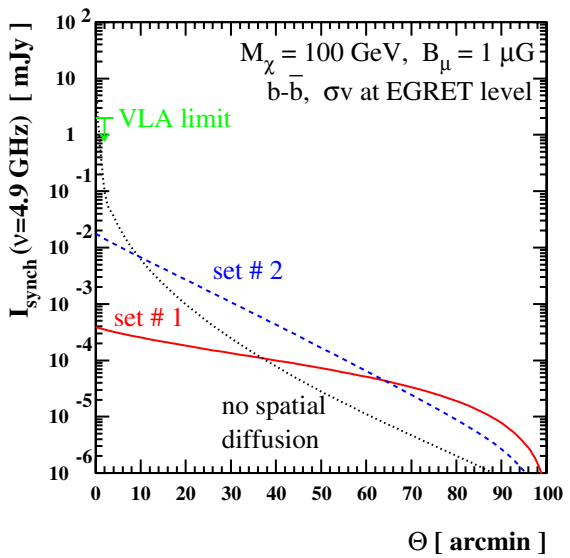

FIG. 12 (color online). Radio flux density spectrum (left panel) and surface brightness distribution at the frequency $\nu=4.9 \mathrm{GHz}$ for a sample WIMP model of mass $100 \mathrm{GeV}$ annihilating into $b \bar{b}$ with a cross section tuned at the level to give 2 events in the EGRET gamma-ray telescope. Results are given for the two choices of propagation parameters already considered in Ref. [11], and for the surface brightness, for illustrative purposes only, in the case when spatial diffusion is neglected. Surface brightness is plotted for a 3 arcsec angular acceptance, corresponding to the VLA angular resolution at the time a search with this instrument for a point source at the center of Draco was performed (the obtained upper limit is plotted in the figure). 

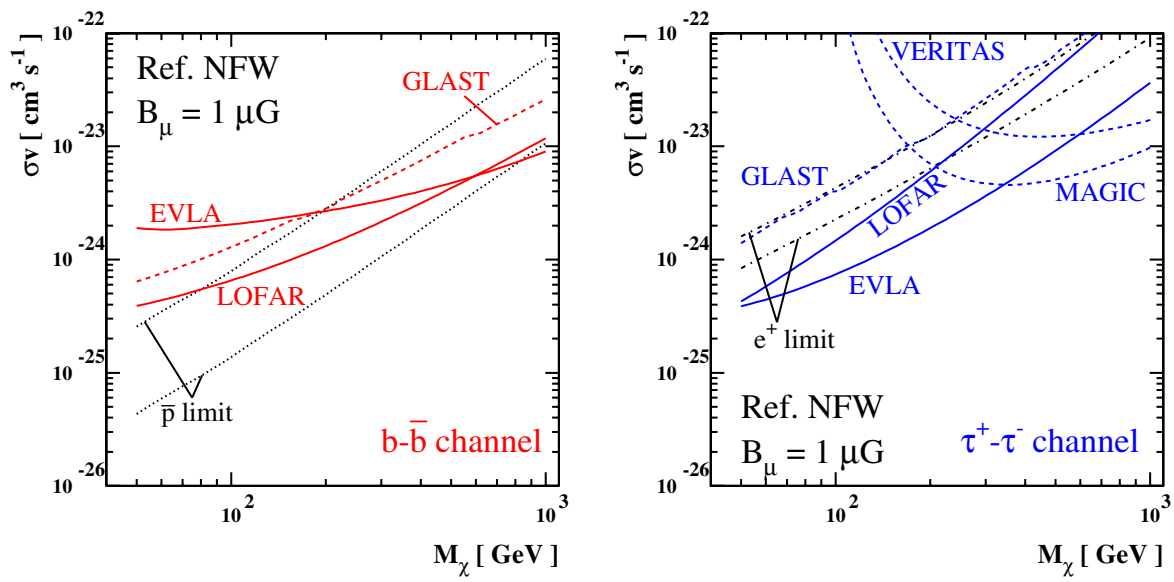

FIG. 13 (color online). The projected sensitivity of future diffuse radio source searches from the direction of Draco, in the same setup as in Fig. 9. For comparison, we also indicate the sensitivity of gamma-ray search experiments, and the constraints from the antiproton and positron fluxes (the two lines corresponding to two different propagation setups for the Milky Way).

over a large angular size, standing clearly as diffuse emission.

No search for a diffuse radio emission from Draco has been performed so far. Even with a future next generation radio telescope the quoted sensitivities do not apply for an extended source. To address the discovery potential of such apparata for the signal we are analyzing, we make a simple extrapolation on the quoted point source sensitivity $I_{p}(\nu)$ for a reference angular resolution $\Delta \Omega_{p}$ and integration time $\Delta t_{p}$, assuming a homogeneous background:

$$
I_{\min }(\nu, \Delta \Omega)=I_{p}(\nu) \frac{\sqrt{\Delta \Omega}}{\sqrt{\Delta \Omega_{p}}} \frac{\sqrt{\Delta t_{p}}}{\sqrt{\Delta t}} .
$$

Reference values for EVLA in phase I [73], at $\nu=5 \mathrm{GHZ}$ are $I_{p}(\nu)=0.8 \mu \mathrm{Jy}$ for $\Delta t_{p}=12 \mathrm{~h}$ and $\Delta \Omega_{p}=$ 0.4 arcsec; for LOFAR observations are all lower frequency up to $\nu=200 \mathrm{MHZ}$, for which $I_{p}(\nu)=0.03 \mathrm{mJy}$ with
$\Delta t_{p}=1 \mathrm{~h}$ and $\Delta \Omega_{p}=0.64 \operatorname{arcsec}[74]$. To infer the projected sensitivity limit, for each WIMP model, halo profile, and frequency of observation, we compute the value for the angular acceptance $\Delta \Omega$ at which $I_{\text {synch }}(\nu, \Delta \Omega) / \sqrt{\Delta \Omega}$ is maximal; we also assume as exposure time $\Delta t=8 \mathrm{hr}$. In Fig. 13 we show results for the sensitivity curves in the plane annihilation cross section versus WIMP mass, for our reference NFW profile, the conservative set \#1 for propagation parameters (set \#2 would give much more favorable results) and a value of the magnetic field equal to $1 \mu \mathrm{G}$. The figure shows that, for this choice of parameters, we predict that WIMP models that are at the level of being detected by GLAST or ACTs in gamma rays, should also give a detectable radio flux, possibly with an even higher sensitivity in a favorable propagation configuration. However, this last conclusion is more model dependent, since some of the parameters are crucial for the estimate of the radio flux. In Fig. 14 we illustrate this point, fixing the
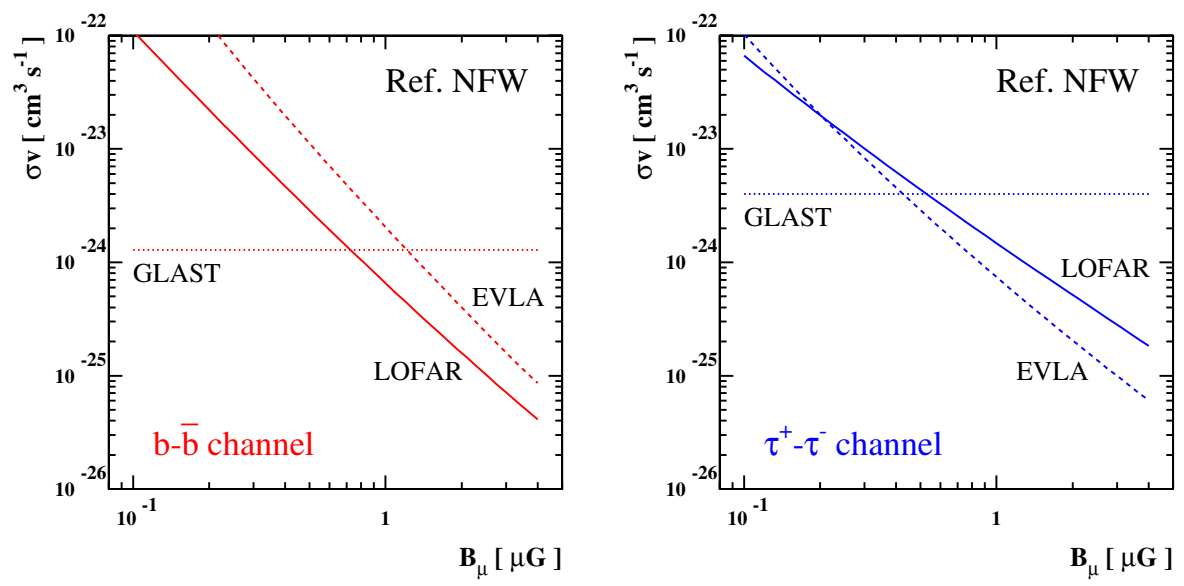

FIG. 14 (color online). The projected sensitivities of future searches for a diffuse radio source as a function of the magnetic field in Draco, in the same setting as in Fig. 9, with a WIMP mass set to $100 \mathrm{GeV}$. For comparison, we also show the projected sensitivity of GLAST. 


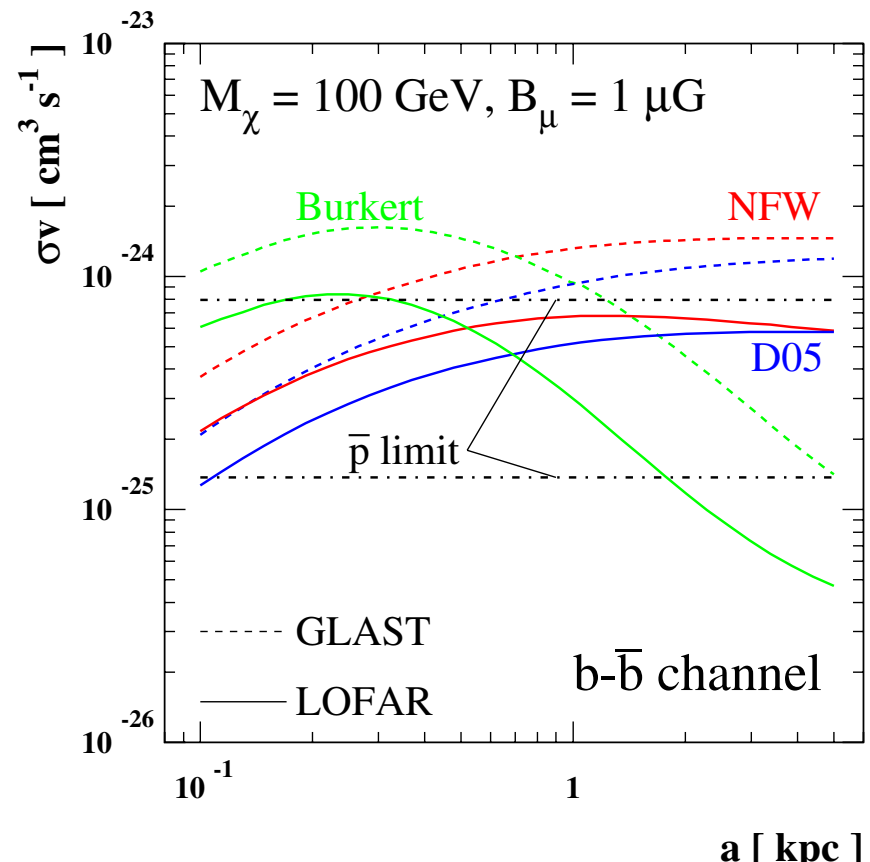

FIG. 15 (color online). LOFAR projected sensitivities, for a given WIMP model and value of the magnetic field in Draco, as a function of the length scale parameter $a$ in the class of halo models selected in Sec. II, compared to the GLAST projected sensitivity.

WIMP mass to $100 \mathrm{GeV}$, varying instead the value of magnetic field in Draco: the trend is obviously that the larger the magnetic field, the higher the induced radio flux, but the dependence is not trivial since the magnetic field enters both in the propagation of electrons and positrons, and in the emission of synchrotron radiation. Finally in Fig. 15 we examine the dependence of the radio sensitivity curves on the model describing the dark matter halo in Draco: we find scalings that are analogous, although different in fine details, to those sketched previously for the gamma-ray fluxes and the corresponding sensitivity of the GLAST satellite.

The inverse Compton emission on the cosmic microwave background and on starlight fills the gap in frequency between radio and gamma-ray frequencies. Let $E=$ $\gamma m_{e} c^{2}$ be the energy of electrons and positrons, $\epsilon$ the target photon energy, and $E_{\gamma}$ the energy of the scattered photon; the inverse Compton power is obtained by folding the differential number density of target photons with the IC scattering cross section:

$$
P_{\mathrm{IC}}\left(E_{\gamma}, E\right)=c E_{\gamma} \int d \epsilon n(\epsilon) \sigma\left(E_{\gamma}, \epsilon, E\right)
$$

where $\sigma\left(E_{\gamma}, \epsilon, E\right)$ is the Klein-Nishina cross section [71] and $n(\epsilon)$ is the differential energy spectrum of the target photons; for simplicity we will assume that the starlight spectrum has the shape of a black body with temperature $T=0.3 \mathrm{eV}$. Such a value of the temperature has been estimated on the basis of the fact that the major part of the Draco star is halo stars somewhat below the turnoff point of the subdwarf main sequence (Odenkirchen et al., 2001). The effective temperature in the HR diagram with $\mathrm{Fe} / \mathrm{H}=-2.0 \mathrm{dex}$ and with an age of $\sim 12 \mathrm{Gyr}$ is of the order of $T \approx 3300-3500 \mathrm{~K}$, which is equivalent to an energy of $\approx 0.28-0.3 \mathrm{eV}$. Folding the IC power with the spectral distribution of the equilibrium number density of electrons and positrons, we get the local emissivity of IC photons of energy $E_{\gamma}$ :

$$
j_{\mathrm{IC}}\left(E_{\gamma}, r\right)=\int d E\left(\frac{d n_{e^{-}}}{d E}+\frac{d n_{e^{+}}}{d E}\right) P_{\mathrm{IC}}\left(E_{\gamma}, E\right)
$$

and the azimuthally averaged surface brightness distribution:

$$
I_{\mathrm{IC}}(\nu, \Theta, \Delta \Omega)=\int_{\Delta \Omega} d \Omega \int_{\text {l.o.s. }} d l \frac{j_{\mathrm{IC}}(\nu, l)}{4 \pi} .
$$

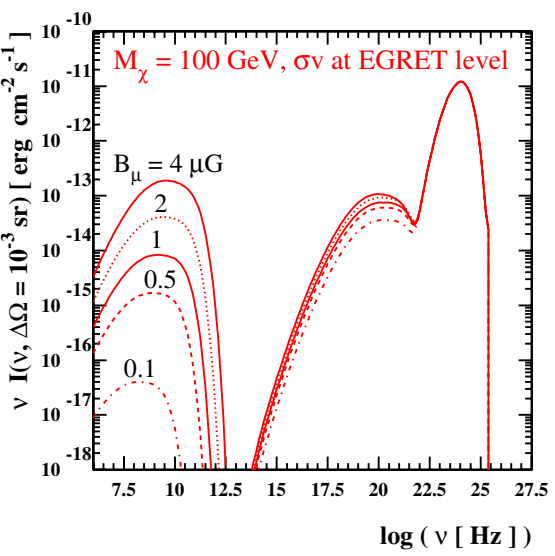

FIG. 16 (color online). Detailed multiwavelength spectrum for a $100 \mathrm{GeV}$ WIMP annihilating into $b-\bar{b}$ (left), and the effect of varying the magnetic field strength. The WIMP pair annihilation rate has been tuned to give a gamma-ray signal at the level of the EGRET measured flux. 
In Fig. 16 we plot the sample multifrequency seed of the emission in Draco due to WIMP annihilations, implementing our reference NFW halo model and reference values for the WIMP mass, the magnetic field, and the various propagation parameters. The WIMP pair annihilation rate has been tuned to give a gamma-ray signal at the level of the EGRET measured flux; the displayed surface brightness is in the direction of the center of Draco and for an angular acceptance equal to the EGRET angular resolution, i.e. not optimized for future observations (we should have in fact considered different solid angles at different wavelengths). As is apparent, there is a significant component in the $\mathrm{x}$-ray band due to inverse Compton on the microwave background radiation, while the contribution on starlight is essentially negligible. The scaling of signals with the assumed value of the magnetic field are also displayed in the right panel.

While so far we have considered simple toy models for the WIMP accounting for the dark matter halo in Draco, in Fig. 17 we consider a few explicit realizations of this scenario within the constrained minimal supersymmetric extension to the standard model (cMSSM), picking among the models studied in [75] those better exemplifying the widest range of possibilities within that particular theoretical setup. All the models are fully consistent with accelerator and other phenomenological constraints, and give a neutralino thermal relic abundance exactly matching the central cosmologically observed value [53]. We adjusted here the values of the universal soft supersymmetry break-

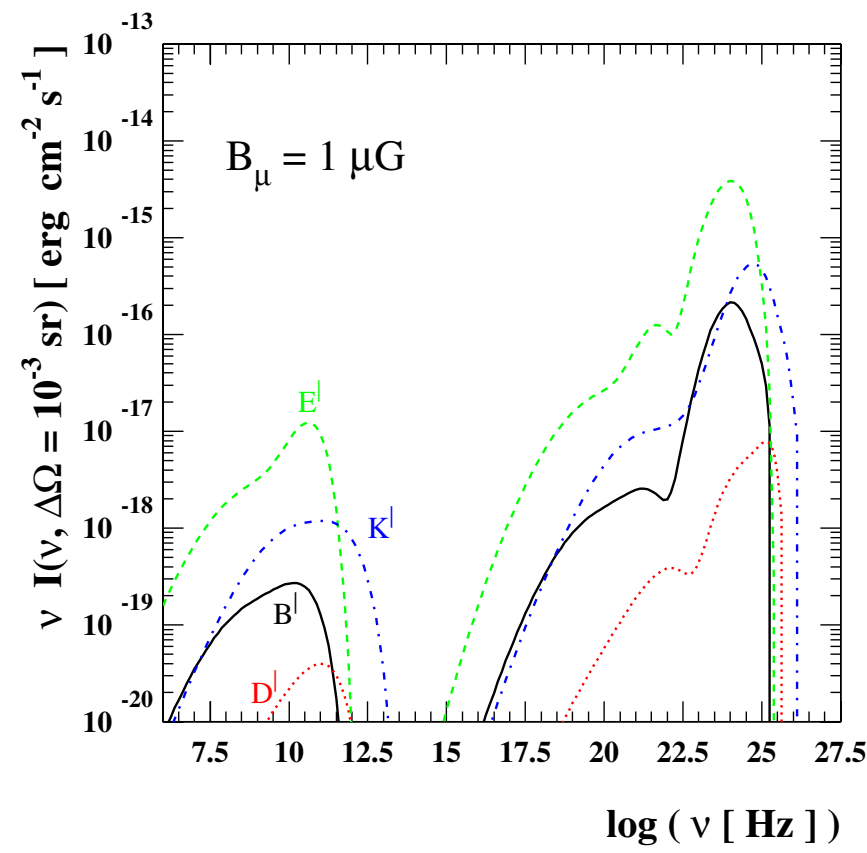

FIG. 17 (color online). Multiwavelength spectra for the four benchmark models described in the text. The prediction is shown for the best-fit NFW profile, and for a mean magnetic field equal to $1 \mu \mathrm{G}$.
TABLE I. The input and output (last two columns, neutralino mass and pair annihilation cross section) parameters of the four cMSSM benchmark models we consider here. Unless otherwise specified, the units for the mass parameters are $\mathrm{GeV}$, and the universal trilinear coupling $A_{0}$ is set to 0 for all models (see [1,75] for details).

\begin{tabular}{lrrrrrrr}
\hline \hline Model & $M_{1 / 2}$ & $m_{0}$ & $\tan \beta$ & $\operatorname{sign}(\mu)$ & $m_{t}$ & $M_{\chi}$ & $(\sigma v) /\left(\mathrm{cm}^{3} \mathrm{~s}^{-1}\right)$ \\
\hline $\mathbf{B}^{\prime}$ (Bulk) & 250 & 57 & 10 & $>0$ & 17594.9 & $7.8 \times 10^{-28}$ \\
$\mathbf{D}^{\prime}$ (Coann.) & 525 & 101 & 10 & $>0$ & 175 & 211 & $8.9 \times 10^{-29}$ \\
$\mathbf{E}^{\prime}$ (Focus P.) & 300 & 1653 & 10 & $>0$ & 171 & 103 & $1.7 \times 10^{-26}$ \\
$\mathbf{K}^{\prime}$ (Funnel) & 1300 & 1070 & 46 & $<0$ & 175 & 554 & $1.1 \times 10^{-26}$ \\
\hline \hline
\end{tabular}

ing scalar mass $m_{0}$ given in [75] in order to fulfill this latter requirement, making use of the latest Isajet v.7.72 release and of the DarkSUSY package [67]. The values of the cMSSM input parameters for the various models are given in Table I (see also Ref. [1]). To facilitate the comparison with other existing analyses, we also list, in the two rightmost columns, the neutralino mass and pair annihilation cross section. Each benchmark model corresponds to a different mechanism responsible for the suppression of the otherwise too large bino relic abundance: $\mathbf{B}^{\prime}$ lies in the bulk region of small supersymmetry breaking masses, and gives a dominant $b-\bar{b}$ final state; $\mathbf{D}^{\prime}$ corresponds to the coannihilation region, and features a large branching ratio for neutralino pair annihilations in $\tau^{+}-\tau^{-} ; \mathbf{E}^{\prime}$ belongs to the focus point region, with a dominant $W^{+}-W^{-}$ final state, and, finally, $\mathbf{K}^{\prime}$ is set to be in the funnel region where neutralinos rapidly annihilate through $s$-channel heavy Higgses exchanges, dominantly producing $b-\bar{b}$ pairs as outcome of annihilations. Not unlike what we found in the case of the multiwavelength analysis of neutralino annihilations in the Coma cluster (see Fig. 25 in Ref. [1]), the most promising among the four benchmark models of Table I is model $\mathbf{E}^{\prime}$, featuring a large pair annihilation cross section to begin with; the less promising model is instead model $\mathbf{D}^{\prime}$, for which the mechanism suppressing the neutralino relic abundance in the early Universe, stau coannihilations, is not associated to pair annihilations of neutralinos today.

Lastly, we found that the SZ effect produced by DM annihilation in Draco, even though a definite probe of the DM annihilation in such cosmic structures (see, e.g., $[9,76])$ is quite low when we take into account the spatial diffusion of secondary electrons: we find, in fact, that the SZ signal towards the center of Draco is negligible even when we normalize the gamma-ray signal at the level of the EGRET upper limit.

\section{A BLACK HOLE AT THE CENTER OF DRACO?}

There is one further effect which could change substantially our picture: if a black hole is present at the center of Draco, and had it formed through an adiabatic accretion process, the ambient dark matter population would have 
experienced a sharp increase in its density profile, turning into a "spike" of dark matter, with a dramatic enhancement in the dark matter annihilation rate at the center of Draco. Such a spike was originally proposed for the Milky Way [77-80] in connection with its central black hole, which has a mass of about $310^{6} M_{\odot}$, and, more recently, it has been extrapolated to small mass halos [81,82], including substructures within the Milky Way dark matter halo, eventually embedding black holes of intermediate mass, in the range between $10^{2}$ to $10^{6} M_{\odot}$.

There is strong observational evidence for the existence of supermassive black holes (in mass range between $10^{6}$ and $10^{9} M_{\odot}$ ), without, however, a detailed understanding on how those objects form, or on the mechanism enforcing the observed correlations with properties of the hosting halos. In one of the proposed scenarios, these two issues are addressed in terms of preexisting intermediate-mass black hole seeds, forming in turn in proto-galaxy environments [83-85]: a significant population of these smaller mass objects would still be present in galaxy-size halos, most likely associated to substructures which have not been tidally disrupted, while merging into the halo. Their presence in the Milky Way halo would be very hard to

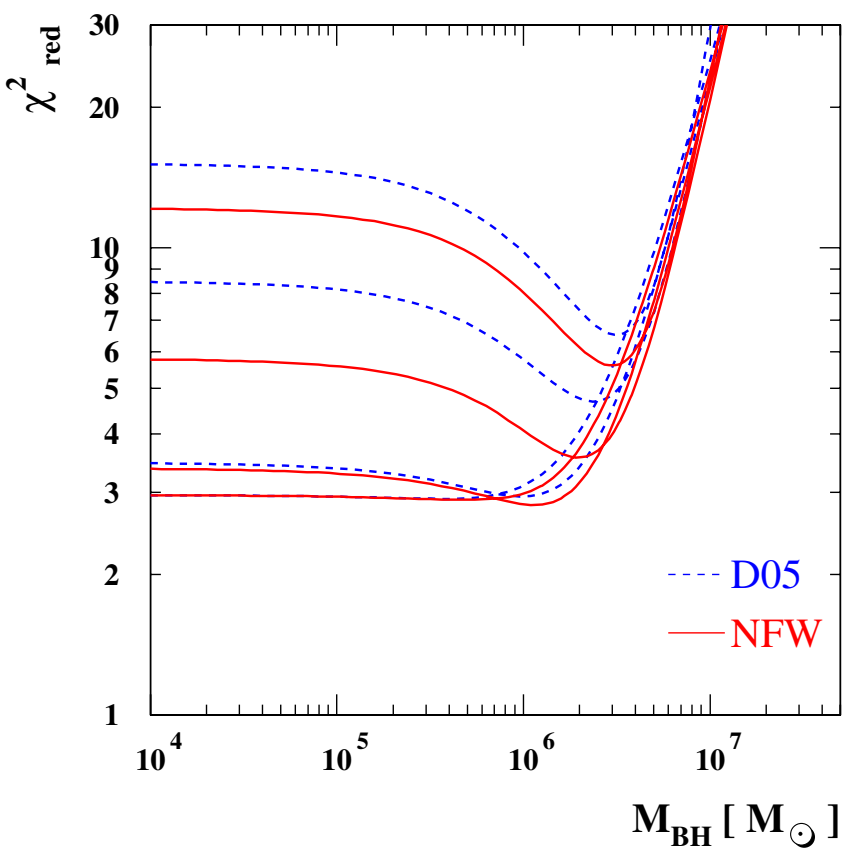

FIG. 18 (color online). Reduced $\chi^{2}$ for a fit of the star velocity dispersion data of Draco in the binning of Munoz et al. under the hypothesis that a black hole of given mass is present at the center of Draco; the dark matter profiles are described by a NFW or a D05 profile with scale factor $a=1 \mathrm{kpc}$ and, respectively, $\rho^{\prime}$ equal to $10^{7}, 2 \times 10^{7}, 3 \times 10^{7}, 3.72 \times 10^{7} M_{\odot} \mathrm{kpc}^{-3}$ (from top to bottom in the figure; the last value corresponds to the best fit in the case without the black hole) and $5 \times 10^{6}, 10^{7}, 2 \times 10^{7}$, $2.54 \times 10^{7} M_{\odot} \mathrm{kpc}^{-3}$ (again, from top to bottom in the figure, with last value being the best fit in the case without the black hole). prove in terms of standard astrophysical observations. In particular, there is no evidence for the presence of a black hole at the center of Draco: it is reasonable to expect that a black hole, being in such a gas poor environment, would be in a dormant phase, rather than in an accreting and luminous one. In Fig. 18 we sketch the dynamical response of adding a black hole of given mass on top of the mass models introduced in Sec. II (the response of the dark matter profile, as specified below, is included): the fit of the star velocity dispersion is not sensitive to black holes of mass smaller than about $10^{5} M_{\odot}$, slightly improves for masses around a few times $10^{6} M_{\odot}$, while the presence of a black hole of mass larger than about $10^{7} M_{\odot}$ is dynamically excluded.

We will take a phenomenological approach and make the hypothesis that a black hole of given mass $M_{B H}$ has formed adiabatically at the center of Draco. The process turns an initial (i.e. before the black hole has accreted the bulk of its mass) dark matter density profile scaling as $\rho_{i}\left(r_{i}\right) \propto r_{i}^{-\gamma}$ into a final profile of the form $\rho_{f}\left(r_{f}\right) \propto r_{f}^{-A}$ : in a simplified system with all dark matter particles on circular orbits, it is easy to show that conservation of mass and angular momentum imply that $A=(9-2 \gamma) /(4-\gamma)$ $[77,78,86]$, i.e. that the final profile is significantly steeper than the initial; this result holds also in a general setup. To derive the right normalization, on the other hand, one has to refer to the full phase space distribution function for the dark matter profile and implement the appropriate adiabatic invariants. We refer here to the procedure outlined in [78]; in the same paper it is shown that, since the growth of the spike depends on the existence of a very large population of cold particles at the center of the dark matter system, where the black hole is adiabatically growing, large spikes form for singular profiles, which embed such a large number of cold states, while it does not for cored profiles for which it is not the case. We will discuss then only the case for the NFW profile and the D05 profile.

In Fig. 19 we plot the line-of-sight integral function $J$, we have introduced in Eq. (1) as a relevant quantity for predictions on the gamma-ray flux, as a function of the black hole mass, and for a given value of the WIMP annihilation cross section $\sigma v$, or vice versa. The value of $\sigma v$ enters critically since the very singular spike density profile has to be extrapolated down to the radius at which a maximal WIMP density is enforced by WIMP pair annihilations, i.e. [77]:

$$
\rho_{\max }=\frac{M_{\chi}}{(\sigma v)\left(t_{0}-t_{f}\right)}
$$

where $t_{0}$ is the present time and $t_{f}$ the formation time of Draco. As can be see, enhancements in the gamma-ray flux of even 4 orders of magnitude are at hand; scalings in black hole mass and $\sigma v$ are analogous for the two halo models considered here. 

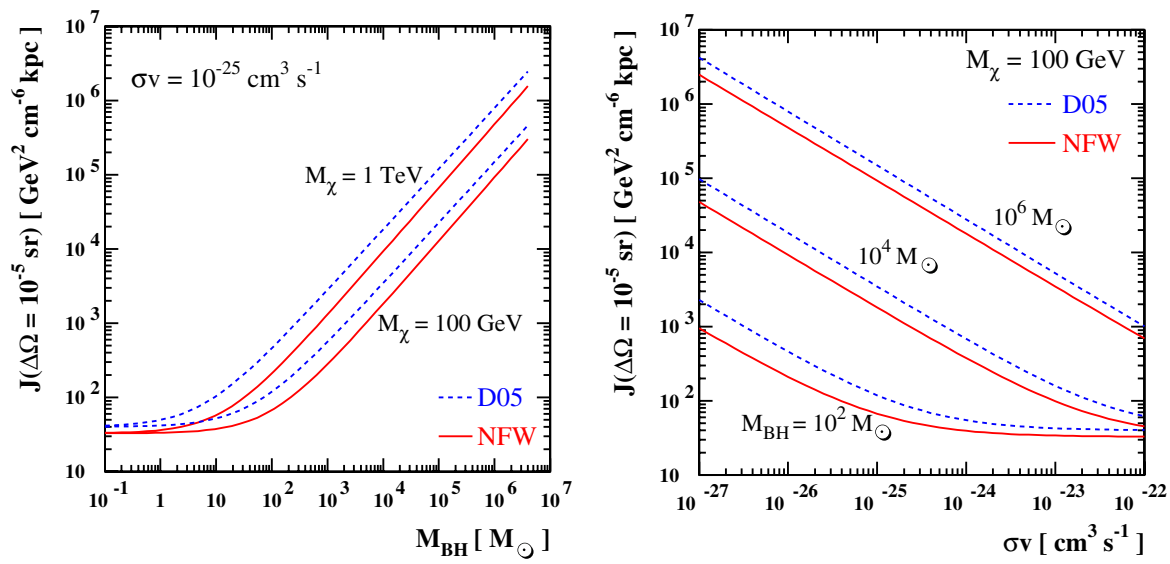

FIG. 19 (color online). Left panel: integral of the square of dark matter density along the line of sight towards the center of Draco and averaged over an angular acceptance of $10^{-5} \mathrm{sr}$, in the case of a central enhancement of the dark matter density due the adiabatic formation at the center of the system of a black hole of given mass; the background dark matter profile are those considered in the left panel and for $\rho^{\prime}$ equal to $3.72 \times 10^{7} M_{\odot} \mathrm{kpc}^{-3}$ (NFW profile) and $2.54 \times 10^{7} M_{\odot} \mathrm{kpc}^{-3}$ (D05 profile). The value of the WIMP pair annihilation cross section and mass enter in the estimate of central maximal WIMP number density set by pair annihilations. Right panel: same as in the left panel but for a few values of the black hole mass and varying the pair annihilation cross section.

The spike is confined in a very tiny portion of the halo, essentially the region within which the black hole dominates the potential well of the final configuration, i.e. smaller than $1 \mathrm{pc}$ even for the heaviest black holes we are considering. The induced gamma-ray source would appear as a point source even at future telescopes with

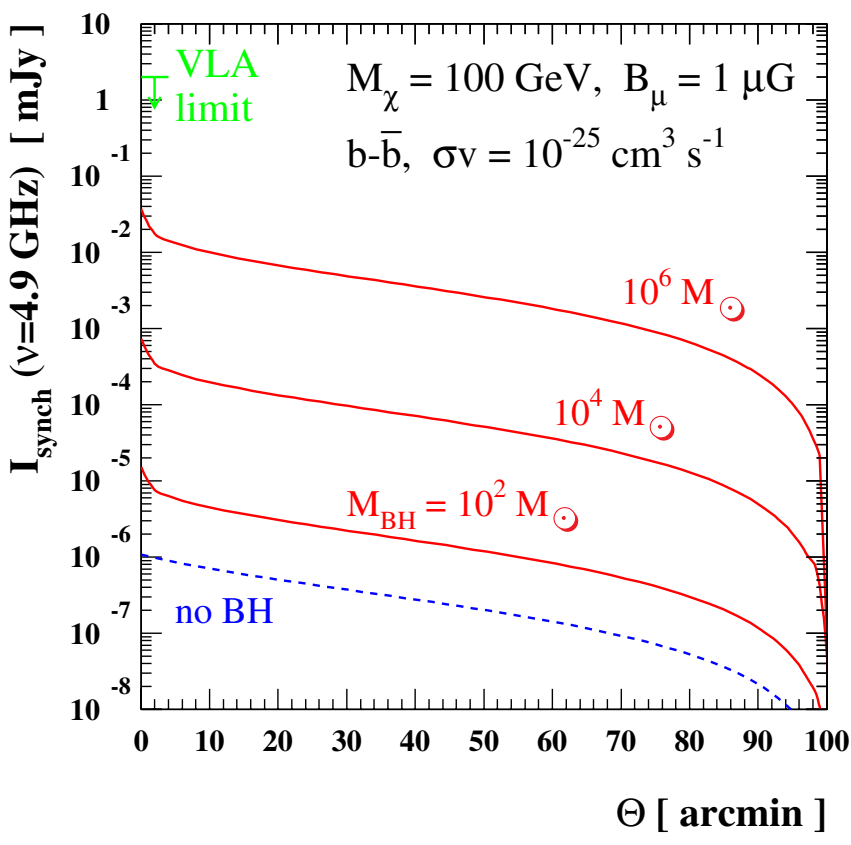

FIG. 20 (color online). We show the radio (at $\nu=4.9 \mathrm{GHz}$ ) surface brightness of Draco in the case of an adiabatic growth of a black hole of given mass $M_{B H}=10^{2}, 10^{4}$, and $10^{6} M_{\odot}$, as labeled. A reference neutralino $b \bar{b}$ model with $M_{\chi}=100 \mathrm{GeV}$ and $\sigma v=10^{-25} \mathrm{~cm}^{3} \mathrm{~s}^{-1}$ with a magnetic field $B_{\mu}=1 \mu \mathrm{G}$ are adopted here. improved angular resolution. On the other hand, analogously to the effect we have already discussed for the standard dark matter halo component, the emitted electrons and positrons diffuse out of the central region and give rise to radio and inverse Compton signals on a very wide angular size. In Fig. 20 we consider, for a few sample masses for the central black hole and one reference annihilation cross section, the induced radio surface brightness in the same configuration displayed in Fig. 12 (propagation parameters in set \#1).

In Fig. 21 we show the scaling of future expected sensitivities in the plane annihilation rate versus black hole mass, for two sample WIMP masses, the $b-\bar{b}$ annihilation channel, the reference NFW halo profile, and set of propagation parameters. As already mentioned above, the effect of the adiabatic black hole growth is more dramatic for WIMP models with smaller annihilation cross section; for comparable annihilation cross section the enhancement in the signal is larger at radio wavelengths than for gamma rays. There is a $1 / 4 \pi$ mismatch since we are essentially adding a point source at the center of the system: we detect only the gamma-rays emitted in our direction, while all emitted electrons and positrons pile up into the population giving rise to the radiation at lower frequencies.

\section{CONCLUSIONS}

Following a program of thorough investigation of the multiwavelength yields of WIMP dark matter annihilations started with the case of the Coma cluster in Ref. [1], in this paper we analyzed the case of the nearby dSph Draco. Under the assumption of equilibrium for the stellar component, we made use of the large wealth of available photometric studies to derive precise mass models for Draco. Under a general setup, we studied the preferred 

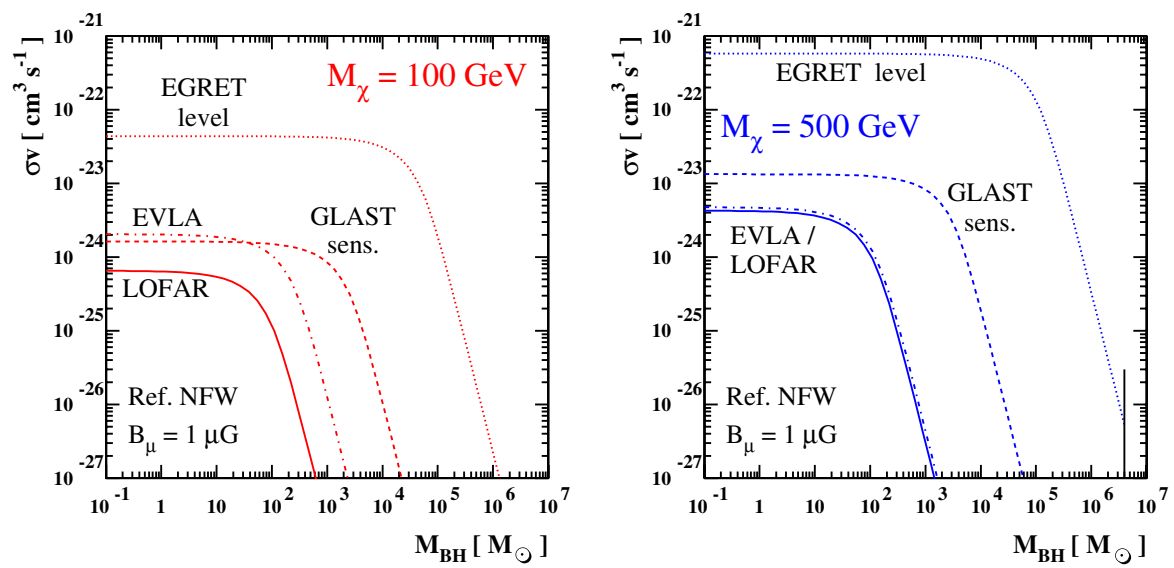

FIG. 21 (color online). We show the projected sensitivity of radio (LOFAR, EVLA) and gamma-ray (EGRET, GLAST) experiments observing Draco as a function of the black hole mass $M_{B H}$ for two choices of the neutralino mass: $M_{\chi}=100 \mathrm{GeV}$ (left panel) and $M_{\chi}=500 \mathrm{GeV}$ (right panel). A reference case of a NFW dark matter profile and a magnetic field $B_{\mu}=1 \mu \mathrm{G}$ are adopted here.

values for the dark matter halo length scale, its density normalization parameter, as well as its anisotropy parameter. Results from numerical simulations of structure formation, together with a proper treatment of the effects of tides on the density profile, enabled us to correlate the concentration parameter and the initial virial mass of the $\mathrm{dSph}$ under consideration. In turn, this allowed us to further constrain the best-fit dark matter halo models for Draco.

We then proceeded to an evaluation of the gamma-ray and electron/positron yield expected from Draco under the hypothesis that the dark matter is in the form of a pairannihilating WIMP. To this extent, we resorted to illustrative cases of WIMPs of given mass and pair-annihilation cross section, dominantly annihilating into final states giving rise to the two extrema of a soft and a hard photon spectrum. For definiteness, and for illustrative purposes, we also considered theoretically well-motivated benchmark supersymmetric models.

We pointed out that unlike the case of the Milky Way galactic center, the spread in the estimate of the gamma-ray flux from Draco is significantly narrow, once the particle physics setup for the dark matter constituent is specified, and that Draco would appear as a pointlike gamma-ray source in both ACTs and GLAST observations. In analogy with our procedure carried out in CPU2006 [1] for larger dark matter halos, we implemented a fully self-consistent propagation setup for positrons and electrons produced in WIMP pair annihilations, and we studied the subsequent generation of radiation in the radio frequencies from synchrotron emissions, and at higher frequencies from inverse Compton scattering off starlight and cosmic microwave background photons.

We showed that, unlike in larger dark matter halos, as is the case for the Coma cluster [1], in small, nearby objects the spatial diffusion of electrons and positrons plays a very significant role. As a consequence, the expected radio emission from Draco is spatially extended, and, depending upon the propagation setup and the values of the magnetic field in Draco, can provide a detectable signal for future radio telescopes. In some cases, we find that an extended radio emission could be detectable from Draco even if no gamma-ray source is identified by GLAST or by ACTs, making this technique the most promising search for dark matter signatures from the class of objects under consideration, i.e. nearby dwarf spheroidal galaxies.

We finally showed that available data can accommodate the presence of a black hole in the center of Draco, even improving the fit to the data for some values of the black hole mass. The corresponding expected enhancement in the gamma-ray flux and in the radio surface brightness for cuspy dark matter halo profiles and an adiabatic growth of the black hole can be of several orders of magnitude. If the mass of the black hole is around or larger than $10^{6} M_{\odot}$, WIMP models are predicted to give unmistakable astrophysical signatures both for future gamma-ray telescopes and for future radio telescopes.
[1] S. Colafrancesco, S. Profumo, and P. Ullio, Astron. Astrophys. 455, 21 (2006).

[2] G. Bertone, D. Hooper, and J. Silk, Phys. Rep. 405, 279 (2005).
[3] A. Cesarini et al., Astropart. Phys. 21, 267 (2004).

[4] L. Bergstrom et al., Phys. Rev. Lett. 94, 131301 (2005).

[5] E. Baltz et al., Phys. Rev. D 65, 063511 (2002).

[6] F. Aharonian et al., Astrophys. J. 636, 777 (2006). 
[7] S. Profumo, Phys. Rev. D 72, 103521 (2005).

[8] S. Colafrancesco and B. Mele, Astrophys. J. 562, 24 (2001).

[9] S. Colafrancesco, Astron. Astrophys. 422, L23 (2004).

[10] E. Giraud et al., Astronomy, Cosmology and Fundamental Physics: Proceedings of the ESO/CERN/ESA Symposium, ESO ASTROPHYSICS SYMPOSIA, edited by P.A. Shaver, L. DiLella, and A. Gimnez (Springer-Verlag, Berlin, 2003), ISBN 3-540-40179-2, p. 448.

[11] M. Mateo, Annu. Rev. Astron. Astrophys. 36, 435 (1998).

[12] G. Tyler, Phys. Rev. D 66, 023509 (2002).

[13] N. W. Evans, F. Ferrer, and S. Sarkar, Phys. Rev. D 69, 123501 (2004).

[14] L. Bergstrom and D. Hooper, Phys. Rev. D 73, 063510 (2006).

[15] S. Profumo and M. Kamionkowski, J. Cosmol. Astropart. Phys. 03 (2006) 003.

[16] Xiao-Jun Bi, Hong-Bo Hu, and Xinmin Zhang, astro-ph/ 0603022.

[17] P. Marleau, in Proceedings of TAUP, Zaragoza, Spain, 2005 (unpublished); M. Tripathi, in Proceedings of Cosmic Rays to Colliders 2005, Prague, Czech Republic, 2005 (unpublished); in Proceedings of $\mathrm{TeV}$ Particle Astrophysics Workshop, Batavia, USA, 2005 (unpublished); M. Chertok, in Proceedings of PANIC 05, Santa Fe, 2005 (unpublished).

[18] L. Wai, Analysis of Draco with EGRET, http://wwwglast.slac.stanford.edu/ScienceWorkingGroups/ DarkMatter/oldstuff/9-9-02.ppt.

[19] V. V. Vassiliev et al., Proceedings of the 28th International Cosmic Ray Conference, Trukuba, Japan, 2003, edited by T. Kajita, Y. Asaoka, A. Kawachi, Y. Matsubara, and M. Sasaki, p. 2851.

[20] E. Fomalont and B. Geldzhaler, Astron. J. 84, 12 (1979).

[21] U. Klein et al., Astron. Astrophys. 255, 49 (1992).

[22] Z. Zang and E. J. A. Meurs, Astrophys. J. 556, 24 (2001).

[23] S. Colafrancesco, in Proceedings of the International Astronomical Union 198, IAU Colloquium, 2005, edited by H. Jerjen and B. Binggeli (Cambridge University Press, Cambridge, England, 2005), p. 229.

[24] D. N. Spergel et al., astro-ph/0603449.

[25] S. Mashchenko, A. Sills, and H.M.P. Couchman, Astrophys. J. 640, 252 (2006).

[26] A. M. Green, S. Hofmann, and D. J. Schwarz, J. Cosmol. Astropart. Phys. 08 (2005) 003.

[27] S. Profumo, K. Sigurdson, and M. Kamionkowski, Phys. Rev. Lett. 97, 031301 (2006).

[28] D. A. C. Lin and S. D. Murray, Astron. Soc. Pac. Conf. Ser. 13, 55 (1991); A. J. Benson, C. S. Frenk, C. G. Lacey, C. M. Baugh, and S. Cole, Mon. Not. R. Astron. Soc. 333, 177 (2002).

[29] D. Reed et al., Mon. Not. R. Astron. Soc. 357, 82 (2005).

[30] F. Stoehr, S. D. M. White, G. Tormen, and V. Springel, Mon. Not. R. Astron. Soc. 335, L84 (2002); J. D. Simon, Bull. Am. Astron. Soc. 36, 1478 (2004).

[31] J. Binney and G. A. Mamon, Mon. Not. R. Astron. Soc. 200, 361 (1982).

[32] E. L. Lokas and G. A. Mamon, Mon. Not. R. Astron. Soc. 343, 401 (2003).

[33] M. Odenkirchen et al., Astron. J. 122, 2538 (2001).

[34] J.L. Sersic, Atlas de Galaxias Australes (Obs. Astron.
Univ. Nac. Cordoba, Cordoba, 1968).

[35] E. L. Lokas and G. A. Mamon, Mon. Not. R. Astron. Soc. 363, 918 (2005).

[36] A. Aparicio, R. Carrera, and D. Martinez-Delgado, Astron. J. 122, 2524 (2001).

[37] M.-R. L. Cioni and H. J. Habing, Astron. Astrophys. 442, 165 (2005).

[38] A. Z. Bonanos, K. Z. Stanek, A. H. Szentgyorgyi, D. D. Sasselov, and G. A. Bakos, Astron. J. 127, 861 (2004).

[39] M.L. Mateo, Annu. Rev. Astron. Astrophys. 36, 435 (1998).

[40] J. F. Navarro, C. S. Frenk, and S. D. M. White, Astrophys. J. 462, 563 (1996); 490, 493 (1997).

[41] J. Diemand, M. Zemp, B. Moore, J. Stadel, and M. Carollo, Mon. Not. R. Astron. Soc. 364, 665 (2005).

[42] A. Burkert, Astrophys. J. 447, L25 (1995).

[43] G. Gentile, A. Burkert, P. Salucci, U. Klein, and F. Walter, Astrophys. J. 634, L145 (2005).

[44] M.D. Weinberg and N. Katz, Astrophys. J. 580, 627 (2002).

[45] A. El-Zant, I. Shlosman, and Y. Hoffman, Astrophys. J. 560, 636 (2001).

[46] S. Mashchenko, H. M.P. Couchman, and J. Wadsley, Nature (London) 442, 539 (2006).

[47] G. Tormen, A. Diaferio, and D. Syer, Mon. Not. R. Astron. Soc. 299, 728 (1998).

[48] E. Hayashi, J. F. Navarro, J. E. Taylor, J. Stadel, and T. Quinn, Astrophys. J. 584, 541 (2003).

[49] S. Kazantzidis, L. Mayer, C. Mastropietro, J. Diemand, J. Stadel, and B. Moore, Astrophys. J. 608, 663 (2004).

[50] A. Klypin, H. Zhao, and R. S. Somerville, Astrophys. J. 573, 597 (2002).

[51] R. R. Munoz et al., Astrophys. J. 631, L137 (2005).

[52] M. I. Wilkinson, J. T. Kleyna, N. W. Evans, G. F. Gilmore, M. J. Irwin, and E. K. Grebel, Astrophys. J. 611, L21 (2004).

[53] D. N. Spergel et al., astro-ph/0603449.

[54] J. S. Bullock et al., Mon. Not. R. Astron. Soc. 321, 559 (2001).

[55] L. Bergstrom and D. Hooper, Phys. Rev. D 73, 063510 (2006).

[56] S. Profumo and M. Kamionkowski, J. Cosmol. Astropart. Phys. 03 (2006) 003.

[57] See www-glast.slac.stanford.edu/software/IS/ glast_lat_performance.htm.

[58] P. Sreekumar et al. (EGRET Collaboration), Astrophys. J. 494, 523 (1998).

[59] P. Majumdar et al., Proceedings of the 29th International Cosmic Ray Conference, Pune, India, 2005, edited by B. Sripathi Acharya, S. Gupta, P. Jagadeesan, A. Jain, S. Karthikeyan, S. Morris, and S. Tonwar (Tata Institute of Fundamental Research, Mumbai, 2005), Vol. 5, p. 203.

[60] H. Krawczynski, D. A. Carter-Lewis, C. Duke, J. Holder, G. Maier, S. Le Bohec, and G. Sembroski, Astropart. Phys. 25, 380 (2006).

[61] L. Bergström, P. Ullio, and J. H. Buckley, Astropart. Phys. 9, 137 (1998).

[62] B. Murakami and J.D. Wells, Phys. Rev. D 64, 015001 (2001); T. Moroi and L. Randall, Nucl. Phys. B570, 455 (2000); M. Fujii and K. Hamaguchi, Phys. Lett. B 525, 143 (2002); Phys. Rev. D 66, 083501 (2002); R. Jeannerot, 
X. Zhang, and R. H. Brandenberger, J. High Energy Phys. 12 (1999) 003; W. B. Lin, D. H. Huang, X. Zhang, and R. H. Brandenberger, Phys. Rev. Lett. 86, 954 (2001); P. Salati, Phys. Lett. B 571, 121 (2003); F. Rosati, Phys. Lett. B 570, 5 (2003); S. Profumo and P. Ullio, J. Cosmol. Astropart. Phys. 11 (2003) 006; R. Catena, N. Fornengo, A. Masiero, M. Pietroni, and F. Rosati, Phys. Rev. D 70, 063519 (2004); M. Kamionkowski and M. S. Turner, Phys. Rev. D 42, 3310 (1990); S. Profumo and P. Ullio, Proceedings of the 39th Rencontres de Moriond Workshop on Exploring the Universe: Contents and Structures of the Universe, La Thuile, Italy, 2004, edited by J. Tran Thanh Van, hep-ph/0305040; G. Gelmini, P. Gondolo, A. Soldatenko, and C. E. Yaguna, Phys. Rev. D 74, 083514 (2006).

[63] S. Orito et al. (BESS Collaboration), Phys. Rev. Lett. 84, 1078 (2000).

[64] J. J. Beatty et al., Phys. Rev. Lett. 93, 241102 (2004).

[65] H. Gast, J. Olzem, and S. Schael, astro-ph/0605254.

[66] P. Picozza and A. Morselli, astro-ph/0604207.

[67] P. Gondolo, J. Edsjo, P. Ullio, L. Bergstrom, M. Schelke, and E. A. Baltz, J. Cosmol. Astropart. Phys. 07 (2004) 008.

[68] S. Profumo and P. Ullio, J. Cosmol. Astropart. Phys. 07 (2004) 006.

[69] A. W. Strong and I. V. Moskalenko, Astrophys. J. 509, 212 (1998); A. W. Strong and I. V. Moskalenko, in Proceedings of the 26th ICRC, Salt Lake City, 1999, AIP Conf. Proc. No. 516 (AIP, New York, 2000), p. 255; in Proceedings of the 27th ICRC, Hamburg, 2001 (Copernicus, Gesellschaft, 2001), p. 1942.

[70] F. Donato, N. Fornengo, D. Maurin, P. Salati, and R.
Taillet, astro-ph/0306312.

[71] M. Longair, High Energy Astrophysics (Cambridge University Press, Cambridge, England, 1994), Vol. 2.

[72] C. Tyler, Phys. Rev. D 66, 023509 (2002).

[73] See www.nrao.edu/evla/townmeeting/rperley.ppt.

[74] H. Rottgering, New Astron. Rev. 47, 405 (2003); H. Rottgering, A. G. de Bruyn, R.P. Fender, J. Kuijpers, M.P. van Haarlem, M. Johnston-Hollitt, and G. K. Miley, astro-ph/0307240.

[75] M. Battaglia, A. De Roeck, J. R. Ellis, F. Gianotti, K. A. Olive, and L. Pape, Eur. Phys. J. C 33, 273 (2004).

[76] T. Culverhouse, W. Evans, and S. Colafrancesco, Mon. Not. R. Astron. Soc. 368, 659 (2006).

[77] P. Gondolo and J. Silk, Phys. Rev. Lett. 83, 1719 (1999).

[78] P. Ullio, H. Zhao, and M. Kamionkowski, Phys. Rev. D 64, 043504 (2001).

[79] D. Merritt, M. Milosavljevic, L. Verde, and R. Jimenez, Phys. Rev. Lett. 88, 191301 (2002).

[80] G. Bertone and D. Merritt, Phys. Rev. D 72, 103502 (2005).

[81] H. S. Zhao and J. Silk, Phys. Rev. Lett. 95, 011301 (2005).

[82] G. Bertone, A. R. Zenter, and J. Silk, Phys. Rev. D 72, 103517 (2005).

[83] R. Islam, J. Taylor, and J. Silk, Mon. Not. R. Astron. Soc. 340, 647 (2003).

[84] M. Volonteri, F. Haardt, and P. Madau, Astrophys. J. 582, 559 (2003).

[85] S. M. Koushiappas, J. S. Bullock, and A. Dekel, Mon. Not. R. Astron. Soc. 354, 292 (2004).

[86] G. D. Quinlan, L. Hernquist, and S. Sigurdsson, Astrophys. J. 440, 554 (1995). 